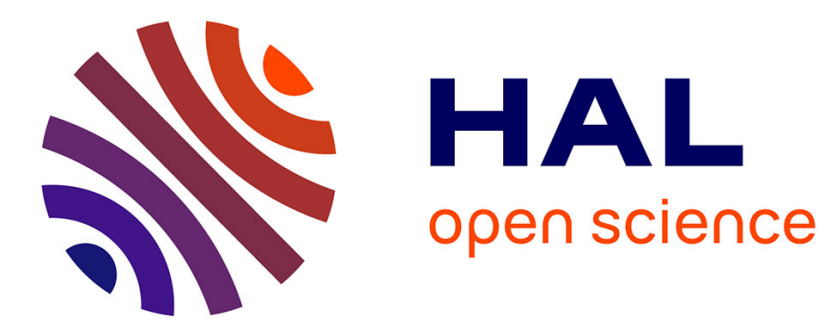

\title{
Idempotent Block Splitting on Partial Partitions, I: Isotone Operators
}

Christian Ronse

\section{To cite this version:}

Christian Ronse. Idempotent Block Splitting on Partial Partitions, I: Isotone Operators. Order, 2011, 28 (2), pp.273-306. 10.1007/s11083-010-9171-3 . hal-02882873

\section{HAL Id: hal-02882873 \\ https://hal.science/hal-02882873}

Submitted on 6 Oct 2020

HAL is a multi-disciplinary open access archive for the deposit and dissemination of scientific research documents, whether they are published or not. The documents may come from teaching and research institutions in France or abroad, or from public or private research centers.
L'archive ouverte pluridisciplinaire HAL, est destinée au dépôt et à la diffusion de documents scientifiques de niveau recherche, publiés ou non, émanant des établissements d'enseignement et de recherche français ou étrangers, des laboratoires publics ou privés. 


\title{
Idempotent block splitting on partial partitions, I: isotone operators
}

\author{
Christian Ronse
}

Received: date / Revised: date

\begin{abstract}
Image segmentation algorithms can be modelled as image-guided operators (maps) on the complete lattice of partitions of space, or on the one of partial partitions (i.e., partitions of subsets of the space). In particular regionsplitting segmentation algorithms correspond to block splitting operators on the lattice of partial partitions, in other words anti-extensive operators that act by splitting each block independently.

This first paper studies in detail block splitting operators and their latticetheoretical and monoid properties; in particular we consider their idempotence (a requirement in image segmentation). We characterize block splitting openings (kernel operators) as operators splitting each block into its connected components according to a partial connection; furthermore, block splitting openings constitute a complete sublattice of the complete lattice of all openings on partial partitions.
\end{abstract}

Our results underlie the connective approach to image segmentation introduced by Serra.

The second paper will study two classes of non-isotone idempotent block splitting operators, that are also relevant to image segmentation.

Keywords partial partitions · complete lattice · block splitting · idempotence $\cdot$ image segmentation

Mathematics Subject Classification (2000) 03E02 - 06B99 - 06A15 . $68 \mathrm{U} 10$

C. Ronse

LSIIT UMR 7005 CNRS-UdS,

Parc d'Innovation, Boulevard Sébastien Brant, BP 10413, 67412 ILLKIRCH CEDEX, FRANCE

E-mail: cronse@unistra.fr, URL: http://lsiit-miv.u-strasbg.fr/ 


\section{Introduction}

In image processing, image segmentation means partitioning space into regions, in such a way that each region is homogeneous (according to some criterion) in the image. We illustrate in Figure 1 three examples of segmentations of an electron micrograph image. Let us explain how this idea can be formalized in terms of partitions.

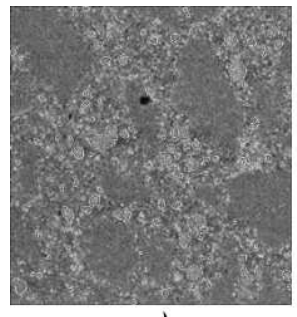

a)

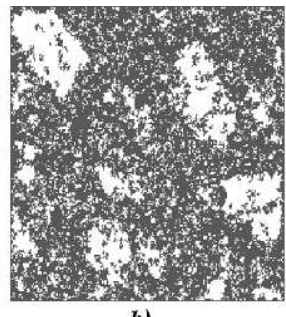

b)
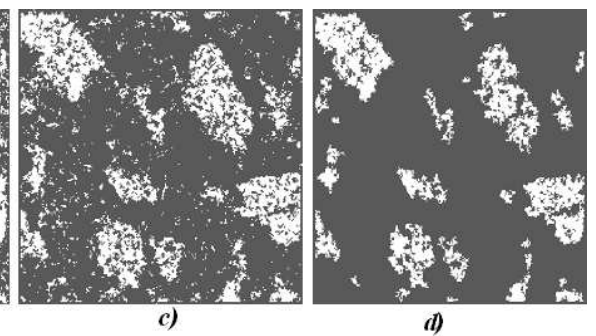

Fig. 1 (From [32].) (a) Electron micrograph of concrete; (b,c,d) are segmentations of (a) by: (b) the "jump" criterion of parameter 12; (c) the "smooth" criterion of parameter 6; (d) the Boolean meet of the two criteria of (b) and (c).

Images can be modeled as functions $E \rightarrow T$, where $E$ is the space of points and $T$ is the set of image values (grey-levels or colours). Write $\mathcal{P}(E)$ for the set of parts of $E$, and $T^{E}$ for the set of all functions $E \rightarrow T$. According to Serra [39], given an image $F: E \rightarrow T$ and a region $A \in \mathcal{P}(E)$ (not necessarily connected), whether $F$ is homogeneous on $A$ or not, depends only on $A$ and on $F$. Thus we have a criterion, that is a map cr: $T^{E} \times \mathcal{P}(E) \rightarrow\{0,1\}$, where for $F: E \rightarrow T$ and $A \in \mathcal{P}(E)$, we have $\operatorname{cr}[F, A]=1$ if $F$ is homogeneous on $A$ according to $\mathrm{cr}$, and $\operatorname{cr}[F, A]=0$ if not; such a criterion can take into account the properties of the restriction of $F$ to $A$ or to its neighbourhood, but also intrinsic properties of $A$ such as shape or size. In practice, most homogeneity criteria require connectedness (in a digital or topological sense), in other words $\operatorname{cr}[F, A]=1$ implies that $A$ is connected. Soille [41] summarizes conventional requirements of segmentation as follows; given an image $F: E \rightarrow T$ :

1. A subset $X$ of $E$ that is not homogeneous for $F$ must be split, leading to a uniquely determined partition $\sigma(X)$ of $X$.

2. The blocks of $\sigma(X)$ are homogeneous (so they should not be split further).

3 . If we merge two (or more) adjacent blocks of $\sigma(X)$, the new merged block is no more homogeneous.

Mathematically speaking, from the criterion $\mathrm{cr}$ and the image $F$, we derive (condition 1 ) a set splitting operator $\sigma$ defined on $\mathcal{P}(E)$, that associates to every $X \in \mathcal{P}(E)$ a partition $\sigma(X)$ of $X$; note that $\sigma$ is uniquely determined by $\mathrm{cr}$ and $F$. From the set splitting operator $\sigma$ one derives the block splitting operator $\beta(\sigma)$ on partitions, that associates to every partition $\pi$ of any set $X$ 
the partition obtained by applying $\sigma$ to each block of $\pi$ separately; in other words if $\pi$ is considered as the set of its blocks, we have $\beta(\sigma)(\pi)=\bigcup_{B \in \pi} \sigma(B)$. For the refinement ordering on partitions [26], we have $\beta(\sigma)(\pi) \leq \pi$. Now condition 2 means that for every $X \in \mathcal{P}(E), \beta(\sigma)(\sigma(X))=\sigma(X)$; equivalently, $\beta(\sigma)$ is idempotent. Then condition 3 means that for every $X \in \mathcal{P}(E)$ and for every partition $\pi$ of $X$ such that $\pi>\sigma(X)$, we have $\beta(\sigma)(\pi)<\pi$; equivalently, given $\pi_{0}$ a partition of some $X \in \mathcal{P}(E)$, for every partition $\pi$ of $X$ such that $\beta(\sigma)\left(\pi_{0}\right)<\pi \leq \pi_{0}$, we must have $\beta(\sigma)(\pi)<\pi$.

Let us now briefly explain how the segmentations of Figure 1 were obtained. A more detailed description is to be found in $[39,32,30]$. We assume that the points are square pixels (i.e., $E \subseteq \mathbf{Z}^{2}$ ), with one of the two usual adjacency relations on pixels, namely the 4-adjacency (horizontal or vertical adjacency) or 8-adjacency (horizontal, vertical or diagonal adjacency); then the neighbourhood of each pixel is a cross of size 5 (in 4-adjacency) or a $3 \times 3$ square (in 8-adjacency); this determines a graph-theoretical connectivity predicate for sets of pixels. In each of the three segmentations $(b, c, d)$, we have a criterion that leads to pixels being painted white or black; then the white connected components, together with the black singletons, form the segmentation partition.

- The segmentation (b) is obtained by the jump algorithm. Given an image $F$, a regional minimum of level $m$ is a connected set $M$ of pixels on which $F$ has constant value $m$, and such that every pixel $q \notin M$ adjacent to some pixel of $M$ must have $F(q)>m$. Given a jump parameter $h>0$, we call a seed any connected set $S$ of pixels such that for some level $m, S$ intersects a regional minimum $M$ of level $m$, and for every $p \in S$ we have $m \leq F(p)<m+h$. We paint in white all seeds, and pixels not belonging to any seed are painted in black. Here we took $h=12$.

- For the segmentation (c) we take a slope parameter $s>0$, and paint in white all pixels $p$ such that the restriction of $F$ to the neighbourhood of $p$ is Lipschitz of parameter $s$ (equivalently, for every pixel $q$ adjacent to $p$, $|F(q)-F(p)| \leq s)$; other pixels are painted in black. Here we took $s=6$.

- In segmentation (d) we take the conjunction of the above two criteria. We paint in white every seed $S$ (for $h$ ) such that the restriction of $F$ to the neighbourhood of any pixel $p \in S$ is Lipschitz of parameter $s$.

These segmentations follow the model of connective segmentation $[39,32,30]$, which imposes the following condition on the criterion cr: for every $F: E \rightarrow T$, the family $\mathcal{C}_{\mathrm{cr}}^{F}$ of all $A \in \mathcal{P}(E)$ such that $\operatorname{cr}[F, A]=1$ is a connection, see Subsection 2.2 ; equivalently $[39,30]$, the set of partitions of $E$ with blocks in $\mathcal{C}_{\mathrm{cr}}^{F}$ is closed under the supremum operation (including the void supremum, i.e., it contains the least partition made of singletons), see also Subsection 3.2. Here the set splitting operator $\sigma$ associates to $X$ the greatest partition of $X$ with blocks in $\mathcal{C}_{\mathrm{cr}}^{F}$; then $\beta(\sigma)$ is idempotent and isotone [30], in other words it is an opening (kernel operator), hence it satisfies the above requirements, in particular $\beta(\sigma)\left(\pi_{0}\right)<\pi \leq \pi_{0} \Rightarrow \beta(\sigma)(\pi)=\beta(\sigma)\left(\pi_{0}\right)$. 
Although the aim of segmentation is the construction of a partition of $E$, in the above mathematical analysis of its requirements we considered partitions of any subset of $E$, in other words families of subsets of $E$ (called blocks) that are non-empty and mutually disjoint (but do not necessarily cover $E$ ). We call such objects partial partitions of $E$ [30]. The refinement order on partitions extends to partial partitions, and they constitute a complete lattice that was studied more than 30 years ago by Czekoslovak mathematicians: Draškovičová $[8,9]$, and to a lesser extent Sturm [42]. The properties of this lattice are summarized in $[30,31]$. As can be seen there, and also throughout this paper, the lattice of partial partitions is mathematically more versatile than the one of partitions. Hence the wider framework of partial partitions is mathematically useful.

Note that partial partitions correspond to partial equivalence relations (i.e., symmetric and transitive binary relations), and the latter have been used in programming semantics (cf. PER models, equilogical spaces) [24,33,1]. Furthermore, universal algebra has studied weak congruences, that is, partial equivalence relations compatible with the algebra operations; they were shown to constitute an algebraic lattice [44,43]; a particular case is that of an algebra without operation, giving the lattice of partial equivalence relations.

There are also practical reasons related to image segmentation justifying the use of partial partitions instead of partitions. We gave some arguments in [30]. But we can also look at the segmentations in Figure 1. In each of the three segmentations (b,c,d), the objects are the white connected components, while black points form the background. Formally, the segmentation is the partition made of all white connected components and all black singletons; but then this partition loses the distinction between a black singleton and a white connected component consisting of an isolated singleton, i.e., between a background point and an object made of a single point. The same loss of information occurs if we consider the black zone as a single block. This leads us to consider that the segmentation has produced in fact a partial partition whose blocks are only the white connected components, while the black points, being outside the support of the partial partition, constitute the "background". Thus Serra's connective segmentation approach [39] has been generalized into the one of partially connective segmentation [32,30], where partitions are replaced by partial partitions, and connections by partial connections.

Therefore, the analysis of image segmentation methods, in particular the three requirements discussed above, leads us to investigate block splitting operators on partial partitions, that is, anti-extensive maps that act by splitting each block independently, and conditions leading to their idempotence. In this respect, what can we gain from previous works ? If we look at the mathematical literature on partitions, after the seminal works of Dubreil [10] and Ore [26], most studies have dealt with conditions for some identities to hold (such as the modular one), geometrical and combinatorial properties of the lattice of partitions of a set of size $n$, etc. The works of Draškovičová $[8,9]$ and Sturm [42] on partial partitions follow the same path. However, the study of operators (maps) on (partial) partitions with given lattice-theoretical or monoid properties, has been largely neglected. The only work we are aware 
of is that of Jordens and Sturm [19,20], who linked Moore families (in other words, closure operators) on partitions with those on sets. In the literature on image segmentation, some operators on partial partitions have been designed, but their algebraic properties were not investigated. This is particularly the case with recent works by Serra [38] and Soille [41], whose operators have in fact very interesting properties that we will analyse in the second paper.

The purpose of this work divided into two parts is the study of block splitting operators on partial partitions, and conditions for their idempotence. Most of the idempotent operators that we obtain follow the constructions given in the framework of image segmentation [38,39,41]; some of them extend to partial partitions some techniques previously used for processing binary or grey-level images [25], that had also been analysed in a general latticetheoretical framework [28]. In fact, the idempotent operators that we obtain are either openings, or order-theoretical generalizations of openings that the author studied long ago under the names of open-condensations [27] and openovercondensations [28].

This work belongs to an ongoing study of lattice-theoretical and monoid properties of maps on partial partitions. It follows a paper analysing adjunctions (residuations) on partitions and partial partitions [31]. Future works will study extensive operators on partial partitions, for example by closing (as in $[19,20]$ ) or clustering blocks; this topic is relevant for region growing approaches to image segmentation.

\subsection{Paper organization}

Subsection 1.2 summarizes our terminology; it follows [31], and mixes classical lattice-theoretical terminology with that used in mathematical morphology (a lattice-based approach in image processing), cf. Table 1; we also recall the definition of basic morphological operators on sets. Then Section 2 gives the mathematical background: first the basic properties of the lattice of partial partitions, then those of partial connections. Section 3 studies block splitting operators on partial partitions. In particular, we show that a block splitting opening is always the decomposition of blocks into their connected components according to a partial connection. Finally Section 4 gives a provisional conclusion to this first paper. We give as appendix a table of our notation (in the order of first appearance).

The second paper will consider idempotent non-isotone block splitting operators, in particular those introduced by Serra [38] and Soille [41].

\subsection{Terminology}

We adopt the terminology of [31], which is a compromise between that of classical lattice theory $[4,6,13,14]$ and that of mathematical morphology $[22,34$, $35,16,40]$, a branch of image processing based on a lattice approach. Indeed, 
mathematical morphology has a rich repertoire of maps with various latticetheoretical and monoid properties, and many of them have no established denomination in classical lattice theory. However the two terminologies sometimes conflict, so we have avoided using confusing terms. Table 1 compares the two terminologies, and gives in italics our choice.

The only order-theoretical structures that we consider are posets and complete lattices; in other words, whenever we mention "the lattice X", this means implicitly "the complete lattice X". Thus our terminology is given in this general framework: a notion involving order only is defined for any poset, while a notion involving suprema and infima is defined for any complete lattice; it is never restricted to the power-set lattice $\mathcal{P}(E)$. For example, many authors speak of a closure operator or a Moore family "on sets" or "on $E$ " (where $E$ is an arbitrary set), but for us they are a closure operator on the poset $\mathcal{P}(E)$ or a Moore family of the complete lattice $\mathcal{P}(E)$.

Note also that in a complete lattice, whenever we consider a "complete" property in relation to arbitrary suprema (resp., infima), this includes also the empty supremum (resp., infimum), in other words the least (resp., greatest) element. This applies in particular to the notions of Moore family and dilation (for suprema), dual Moore family and erosion (for infima), complete sublattice and complete morphism (for both suprema and infima).

Let us now introduce our general notation; specific notation introduced in our work is summarized in a table at the end of this paper. Throughout, we consider a "space" $E$, whose elements are called "points"; in fact $E$ is an arbitrary set of size at least 2, although in practice $E$ will be the Euclidean space $\mathbf{R}^{n}$, the digital space $\mathbf{Z}^{n}$, or a bounded interval in such spaces. Points of $E$ will be written $p, q, r, \ldots$, while subsets of $E$ will be designated by uppercase letters $A, B, \ldots, Y, Z$ (except the empty set $\emptyset$ ). Partial partitions of $E$ will be written $\pi, \pi^{\prime}, \pi_{1}, \pi^{1}, \ldots$. Given a set $T$ of values, functions $E \rightarrow T$ will be written $F, G, H, \ldots$.

An abstract poset (or complete lattice) will be written $L, M, \ldots$, and its elements will be denoted with lower-case letters $a, b, \ldots, y, z$, except the least and greatest elements written $\mathbf{0}$ and $\mathbf{1}$ respectively; subsets of $L$ will be designated by upper-case letters $A, B, \ldots, Y, Z$; we write $\leq$ for the order on the poset.

Given two sets $A$ and $B$, we will write $(\alpha, \beta): A \rightleftharpoons B$, or say that $(\alpha, \beta)$ is $A \rightleftharpoons B$, if $\alpha$ is a map $A \rightarrow B$ and $\beta$ is map $B \rightarrow A$.

Given two complete lattices $L$ and $M$ (equal or different), a map $L \rightarrow M$ is called an operator. Operators will be designated by lower-case Greek letters $\alpha, \ldots, \omega$ (except $\pi$, reserved for partial partitions). We generally use $\gamma$ for an opening and $\varphi$ for a closure. We write $\psi(x)$ for the image of $x$ by $\psi$ (for example [14] writes $x \psi$ ); thus the composition of operators is read from right to left: given $\psi: L \rightarrow M$ and $\xi: M \rightarrow N$, the composition of $\psi$ followed by $\xi$ is $\xi \psi: L \rightarrow N: x \mapsto \xi(\psi(x))$. The set $M^{L}$ of operators $L \rightarrow M$, with componentwise order: $\psi \leq \xi$ iff $\psi(x) \leq \xi(x)$ for all $x \in L$, is a complete lattice with componentwise supremum and infimum: $\left[\bigvee_{i \in I} \psi_{i}\right](x)=\left[\bigvee_{i \in I} \psi_{i}(x)\right]$, 
Table 1 Morphological and alternative terminology for lattice-theoretical notions (in italics, the terminology used in this paper)

\begin{tabular}{|c|c|c|}
\hline Morphological & Other & Meaning \\
\hline lower set $[13]$ & down-set [4] & $S: \quad y \leq x \in S \Rightarrow y \in S$ \\
\hline upper set $[13]$ & up-set $[4]$ & $S: \quad y \geq x \in S \Rightarrow y \in S$ \\
\hline $\begin{array}{l}\text { inf-closed family, } \\
\text { Moore family [3] }\end{array}$ & $\begin{array}{l}\text { closure system }[13] \text {, } \\
\text { closure subset }[4], \\
\text { closure range }\end{array}$ & $F: \quad X \subseteq F \Rightarrow \bigwedge X \in F$ \\
\hline $\begin{array}{l}\text { sup-closed family, } \\
\text { dual Moore family [3] }\end{array}$ & $\begin{array}{l}\text { kernel system, } \\
\text { dual closure subset [4] }\end{array}$ & $F: \quad X \subseteq F \Rightarrow \bigvee X \in F$ \\
\hline (not defined) & directed subset $[13]$ & $\begin{array}{l}D: \quad p, q \in D \Rightarrow \\
\quad \exists r \in D, p, q \leq r\end{array}$ \\
\hline operator $[16]$ & $\begin{array}{l}\text { map [6], mapping [4], } \\
\text { function [13] }\end{array}$ & $\psi: L \rightarrow M$ \\
\hline increasing $[22,34]$ & isotone $[4]$ & $\psi: \quad x \leq y \Rightarrow \psi(x) \leq \psi(y)$ \\
\hline decreasing $[16]$ & antitone [4] & $\psi: \quad x \leq y \Rightarrow \psi(x) \geq \psi(y)$ \\
\hline dilation $[35]$ & complete join-morphism & $\delta: \quad \delta\left(\bigvee_{i \in I} x_{i}\right)=\bigvee_{i \in I} \delta\left(x_{i}\right)$ \\
\hline erosion $[35]$ & $\begin{array}{l}\text { complete meet-morphism } \\
\text { complete morphism }\end{array}$ & $\begin{array}{l}\varepsilon: \quad \varepsilon\left(\bigwedge_{i \in I} x_{i}\right)=\bigwedge_{i \in I} \varepsilon\left(x_{i}\right) \\
\text { both dilation and erosion }\end{array}$ \\
\hline adjunction [13] & residuation $[4]$ & $(\varepsilon, \delta): \quad \delta(x) \leq y \Leftrightarrow x \leq \varepsilon(y)$ \\
\hline lower adjoint [13] & residuated [4] & $\delta: \quad(\varepsilon, \delta)$ adjunction \\
\hline upper adjoint [13] & residual $[4]$ & $\varepsilon: \quad(\varepsilon, \delta)$ adjunction \\
\hline extensive $[2,22]$ & increasing [6] & $\psi: \quad \psi(x) \geq x$ \\
\hline anti-extensive $[22]$ & $\begin{array}{l}\text { intensive, contracting, } \\
\text { decreasing }\end{array}$ & $\psi: \quad \psi(x) \leq x$ \\
\hline \multicolumn{2}{|c|}{ idempotent } & $\psi: \quad \psi(\psi(x))=\psi(x)$ \\
\hline closing $[22]$ & closure $[4]$ & $\varphi: \quad x \leq \varphi(y) \Leftrightarrow \varphi(x) \leq \varphi(y)$ \\
\hline opening $[22]$ & $\begin{array}{l}\text { dual closure [4], } \\
\text { kernel operator [13] }\end{array}$ & $\gamma: \quad x \geq \gamma(y) \Leftrightarrow \gamma(x) \geq \gamma(y)$ \\
\hline invariance domain & fixpoint set & $\{x \in L \mid \psi(x)=x\}$ \\
\hline identity & identity operator & id : $x \mapsto x$ \\
\hline \multicolumn{2}{|c|}{ monoid of operators $L \rightarrow L$} & $\begin{array}{l}\mathrm{M} \subseteq L^{L}: \quad \mathbf{i d} \in \mathrm{M} \\
\psi, \xi \in \mathrm{M} \Rightarrow \psi \xi \in \mathrm{M}\end{array}$ \\
\hline
\end{tabular}

and similarly for $\Lambda$. The isotone operators constitute a complete sublattice of the lattice of operators.

When an operator is $L \rightarrow L$, we say that it is "on $L$ ". The set of operators on $L$, with the law of composition, is a monoid (i.e., composition is associative and admits the identity operator as neutral element), and the set of isotone operators is a sub-monoid of it. The invariance domain of an operator $\psi: L \rightarrow$ $L$ is the set $\operatorname{lnv}(\psi)=\{x \in L \mid \psi(x)=x\}$. Recall that a monoid morphism is a map from a monoid to another one (or to itself), that is compatible with the composition law, and that preserves the neutral element.

Let us finally make some remarks on the various notions given in Table 1 . The definition of closure given there, namely for all $x, y \in L, x \leq \varphi(y) \Leftrightarrow$ $\varphi(x) \leq \varphi(y)$, is equivalent to the classical one: $\varphi$ is an isotone, extensive and 
idempotent operator on $L$. For a recent survey on closures, see [11]. Dually, the definition given for an opening, namely $x \geq \gamma(y) \Leftrightarrow \gamma(x) \geq \gamma(y)$, is equivalent to the usual one: $\gamma$ is an isotone, anti-extensive and idempotent operator.

A Moore family or dual Moore family is itself a complete lattice for the order $\leq$. Note that a subset $F$ of $L$ is a Moore family iff it is the invariance domain of a closure, while it is a dual Moore family iff it is the invariance domain of an opening. The set of Moore families (resp., dual Moore families) of $L$ is closed under intersection in $\mathcal{P}(L)$. In the lattice of operators on $L$, the set of closures is closed under infima and the set of openings is closed under suprema. The map $\psi \mapsto \operatorname{lnv}(\psi)$ gives a dual isomorphism between the complete lattice of Moore families and the one of closures, and an isomorphism between the complete lattice of dual Moore families and the one of openings: for two closures $\varphi, \varphi^{\prime}$ and two openings $\gamma, \gamma^{\prime}$, we have

$$
\varphi \leq \varphi^{\prime} \Longleftrightarrow \operatorname{lnv}(\varphi) \supseteq \operatorname{lnv}\left(\varphi^{\prime}\right) \quad \text { and } \quad \gamma \leq \gamma^{\prime} \Longleftrightarrow \operatorname{lnv}(\gamma) \subseteq \operatorname{lnv}\left(\gamma^{\prime}\right) \text {. }
$$

A dilation $\delta$ must satisfy $\delta(\mathbf{0})=\mathbf{0}$, while an erosion must satisfy $\varepsilon(\mathbf{1})=\mathbf{1}$. Dilations and erosions are isotone. In an adjunction $(\varepsilon, \delta), \varepsilon$ is an erosion and $\delta$ is a dilation, $\delta \varepsilon \delta=\delta, \varepsilon \delta \varepsilon=\varepsilon, \delta \varepsilon$ is an opening and $\varepsilon \delta$ is a closure [13,16]. Conversely, given a dilation $\delta: L \rightarrow M$, there is a unique erosion $\varepsilon: M \rightarrow L$ such that $(\varepsilon, \delta)$ is an adjunction, and given an erosion $\varepsilon: M \rightarrow L$, there is a unique dilation $\delta: L \rightarrow M$ such that $(\varepsilon, \delta)$ is an adjunction. Note that $(\varepsilon, \delta)$ is an adjunction iff $\delta$ and $\varepsilon$ are isotone, $\delta \varepsilon$ is anti-extensive and $\varepsilon \delta$ is extensive. The set of adjunctions constitutes a dual isomorphism between the Moore family of erosions and the dual Moore family of dilations.

Let us now recall the basic morphological operators on sets. Our terminology and notation follows that of $[3,16]$, contradicting slightly that of $[22,34,35$, 40]. We suppose that the space $E$ is Euclidean $\left(E=\mathbf{R}^{n}\right)$ or digital $\left(E=\mathbf{Z}^{n}\right)$. For every $p \in E$, the translation by $p$ is the map $E \rightarrow E: x \mapsto x+p$; it transforms any subset $X$ of $E$ into its translate by $p, X_{p}=\{x+p \mid x \in X\}$. Then the Minkowski addition $\oplus[23]$ and Minkowski subtraction $\ominus[15]$ are defined as follows: for any $X, B \in \mathcal{P}(E)$ we set

$$
\begin{aligned}
& X \oplus B=\bigcup_{b \in B} X_{b}=\bigcup_{x \in X} B_{x}=\{x+b \mid x \in X, b \in B\} ; \\
& X \ominus B=\bigcap_{b \in B} X_{-b}=\left\{p \in E \mid B_{p} \subseteq X\right\} .
\end{aligned}
$$

We define then the two operations $\circ$ and $\bullet$ by

$$
\text { and } \quad \begin{aligned}
X \bullet B & =(X \ominus B) \oplus B=\bigcup\left\{B_{p} \mid p \in E, B_{p} \subseteq X\right\} \\
\text { an } & =(X \oplus B) \ominus B .
\end{aligned}
$$

This leads then to four operators on $\mathcal{P}(E)$ : the dilation by $B, \delta_{B}: X \mapsto X \oplus B$; the erosion by $B, \varepsilon_{B}: X \mapsto X \ominus B$; the opening by $B, \gamma_{B}=\delta_{B} \varepsilon_{B}: X \mapsto X \circ B$; the closing by $B, \varphi_{B}=\varepsilon_{B} \delta_{B}: X \mapsto X \bullet B$. The set $B$ is called the structuring element. Clearly, $\left(\varepsilon_{B}, \delta_{B}\right)$ is an adjunction, $\delta_{B}$ is a dilation, $\varepsilon_{B}$ is an erosion, $\gamma_{B}$ is an opening and $\varphi_{B}$ is a closure. 


\section{Background}

Subsection 2.1 recalls basic facts $[8,9,30,31]$ about the lattice of partial partitions. Then Subsection 2.2 recalls the definition and basic properties of partial connections and of the lattice that they constitute [30].

\subsection{The lattice of partial partitions}

We summarize from $[30,31]$ the essential facts about partial partitions and the complete lattice that they make; we follow the same terminology and notation. See also $[8,9]$ for further results.

Every binary relation $R$ on the space $E$ can be identified with the set of ordered pairs $(x, y) \in E^{2}$ such that $x R y$; the support of $R$ is the subset $\operatorname{supp}(R)$ of $E$ comprising all $p \in E$ such that there is some $q \in E$ with $p R q$ or $q R p$. The support of a family $\mathcal{B}$ of subsets of $E$ is the $\operatorname{set} \operatorname{supp}(\mathcal{B})=\bigcup \mathcal{B}$ comprising all points covered by at least one element of $\mathcal{B}$.

A partial equivalence on $E$ is a binary relation on $E$ that is symmetric and transitive. Equivalently, it is a relation that forms an equivalence relation on its support. A partial equivalence is an equivalence relation iff it is reflexive, iff its support is $E$. A partial partition of $E$ is a family $\pi$ of subsets of $E$ that are non-empty and mutually disjoint, in other words, such that every point of $E$ belongs to at most one member of $\pi$. Equivalently, $\pi$ is a partition of its $\operatorname{support} \sup (\pi)$. Every member of a partial partition is called a block [26]. A partial partition is a partition of $E$ iff its support is $E$. There is a natural oneto-one correspondence between partial partitions of $E$ and partial equivalences on $E$; write $\operatorname{PE}(\pi)$ for the partial equivalence on $E$ corresponding to a partial partition $\pi$ of $E$; then we have $\operatorname{supp}(\operatorname{PE}(\pi))=\operatorname{supp}(\pi)$.

With every partial partition $\pi$ is associated the partial partition class map $\mathrm{Cl}_{\pi}: E \rightarrow \mathcal{P}(E)$ given by $\mathrm{Cl}_{\pi}(p)=\emptyset$ if $p \notin \operatorname{supp}(\pi)$, while for $p \in \operatorname{supp}(\pi)$, $\mathrm{Cl}_{\pi}(p)$ is the unique block of $\pi$ to which $p$ belongs; then $\mathrm{Cl}_{\pi}(p)$ is called the class of $p$ in $\pi$. See [30] for a characterization of partial partition class maps. Now the partial equivalence relation $\operatorname{PE}(\pi)$ corresponding to $\pi$ satisfies:

$$
\forall p, q \in E, \quad p \operatorname{PE}(\pi) q \quad \Longleftrightarrow \quad q \in \mathrm{Cl}_{\pi}(p) .
$$

Write $\Pi(E)$ for the set of all partitions of $E$, and $\Pi^{*}(E)$ for the set of all partial partitions of $E$. We have $\Pi^{*}(E)=\bigcup_{A \in \mathcal{P}(E)} \Pi(A)$. Now $\Pi(\emptyset)=\Pi^{*}(\emptyset)$ has a unique element, the empty partition having no block, we write it $\emptyset$. Then $\emptyset \in \Pi^{*}(E)$, and for every $p \in E$ we have $C_{\varnothing}(p)=\emptyset$. Formally, $\emptyset$ is identical to the empty set $\emptyset$, but we use a slightly modified notation in order to distinguish the two roles of the empty set, as least element $\emptyset$ of the lattice $\mathcal{P}(E)$, and as least element $\varnothing$ of the lattice $\Pi^{*}(E)$. For $A \in \mathcal{P}(E)$, let $\mathbf{0}_{A}$ be the partition of $A$ into its singletons, and $\mathbf{1}_{A}$ the partition of $A$ into a single block (or no block if $A=\emptyset$ ):

$$
\mathbf{0}_{A}=\{\{p\} \mid p \in A\} \quad \text { and } \quad \mathbf{1}_{A}=\{A\} \backslash\{\emptyset\}=\left\{\begin{array}{ll}
\{A\} & \text { if } A \neq \emptyset \\
\varnothing & \text { if } A=\emptyset
\end{array} .\right.
$$


Following [26], we call $\mathbf{0}_{A}$ the identity partition of $A$ and $\mathbf{1}_{A}$ the universal partition of $A$. Note that $\mathbf{0}_{\emptyset}=\mathbf{1}_{\emptyset}=\emptyset$.

The well-known refinement ordering on partitions [26] extends to partial partitions. Given $\pi_{1}, \pi_{2} \in \Pi^{*}(E)$, we say that $\pi_{1}$ is finer than $\pi_{2}$, or that $\pi_{2}$ is coarser than $\pi_{1}$, and write $\pi_{1} \leq \pi_{2}$ (or $\pi_{2} \geq \pi_{1}$ ), iff every block of $\pi_{1}$ is included in a block of $\pi_{2}$ :

$$
\pi_{1} \leq \pi_{2} \quad \Longleftrightarrow \quad \forall C_{1} \in \pi_{1}, \exists C_{2} \in \pi_{2}, C_{1} \subseteq C_{2} .
$$

The bijections $\pi \leftrightarrow \mathrm{PE}(\pi) \leftrightarrow \mathrm{Cl}_{\pi}$ between partial partitions, partial equivalences and partial partition class maps induces a one-to-one correspondence between the refinement order on partial partitions, the inclusion order on partial equivalences, and the inclusion of class maps: $\forall \pi_{1}, \pi_{2} \in \Pi^{*}(E)$,

$$
\pi_{1} \leq \pi_{2} \Longleftrightarrow \mathrm{PE}\left(\pi_{1}\right) \subseteq \mathrm{PE}\left(\pi_{2}\right) \Longleftrightarrow \forall p \in E, \mathrm{Cl}_{\pi_{1}}(p) \subseteq \mathrm{Cl}_{\pi_{2}}(p)
$$

Therefore partial partitions, ordered by refinement, constitute a complete lattice isomorphic to the one of partial equivalences. Write $\bigvee$ and $\Lambda$ for the supremum and infimum operations on the complete lattice $\left(\Pi^{*}(E), \leq\right)$. The least (finest) and greatest (coarsest) partial partitions are $\varnothing$ and $\mathbf{1}_{E}$. Given a family $\left\{\pi_{i} \mid i \in I\right\}$ of partial partitions, the class map of their infimum $\bigwedge_{i \in I} \pi_{i}$ is given by intersection of the respective class maps:

$$
\forall p \in E, \quad \mathrm{Cl}_{\bigwedge_{i \in I} \pi_{i}}(p)=\bigcap_{i \in I} \mathrm{Cl}_{\pi_{i}}(p) .
$$

The class map of their supremum $\bigvee_{i \in I} \pi_{i}$ is given by chaining [26] class maps: for $p, q \in E, q \in \mathrm{Cl}_{\bigvee_{i \in I} \pi_{i}}(p)$ iff there is some integer $n \geq 1$ and a sequence $x_{0}, \ldots, x_{n}$ in $E$ with $x_{0}=p$ and $x_{n}=q$, such that for each $t=1, \ldots, n$ there is some $i(t) \in I$ with $x_{t} \in \mathrm{Cl}_{\pi_{i(t)}}\left(x_{t-1}\right)$. Note that (3) and the chaining construction are also valid for $I$ empty: the empty infimum gives as point class the empty intersection, that is, $\mathrm{Cl}_{\mathbf{1}_{E}}(p)=E$, while chaining in an empty family of partitions does not give any point, and we get $\mathrm{Cl}_{\varnothing}(p)=\emptyset$.

Given a family $\mathcal{B}$ of non-empty subsets of $E$ and two points $p, q \in E$, we say that $p$ and $q$ are chained by $\mathcal{B}$ if there are $B_{1}, \ldots, B_{n} \in \mathcal{B}(n \geq 1)$ such that $p \in B_{1}, q \in B_{n}$ and for $i=2, \ldots, n$ we have $B_{i-1} \cap B_{i} \neq \emptyset$. Then in a supremum $\bigvee_{i \in I} \pi_{i}$ of partial partitions, two points $p, q \in E$ belong to the same block iff they are chained by $\bigcup_{i \in I} \pi_{i}$, see Figure 2 .

Note that when the partial partitions have pairwise disjoint supports $(i \neq$ $\left.j \Rightarrow \operatorname{supp}\left(\pi_{i}\right) \cap \operatorname{supp}\left(\pi_{j}\right)=\emptyset\right)$, their supremum is their union: $\bigvee_{i \in I} \pi_{i}=$ $\bigcup_{i \in I} \pi_{i}$. In particular, for any $\pi \in \Pi^{*}(E)$ we have $\pi=\bigcup_{C \in \pi} \mathbf{1}_{C}=\bigvee_{C \in \pi} \mathbf{1}_{C}$.

For $A \in \mathcal{P}(E)$, the non-empty supremum and infimum operations in $\Pi^{*}(A)$ are inherited from $\Pi^{*}(E)$; in other words for a non-void $\left\{\pi_{i} \mid i \in I\right\} \subseteq \Pi^{*}(A)$, $\bigvee_{i \in I} \pi_{i}$ and $\bigwedge_{i \in I} \pi_{i}$ are the same in $\Pi^{*}(A)$ and in $\Pi^{*}(E)$.

A partial partition on $E$ is a partition iff it majorates $\mathbf{0}_{E}$ :

$$
\Pi(E)=\left\{\pi \in \Pi^{*}(E) \mid \pi \geq \mathbf{0}_{E}\right\} .
$$




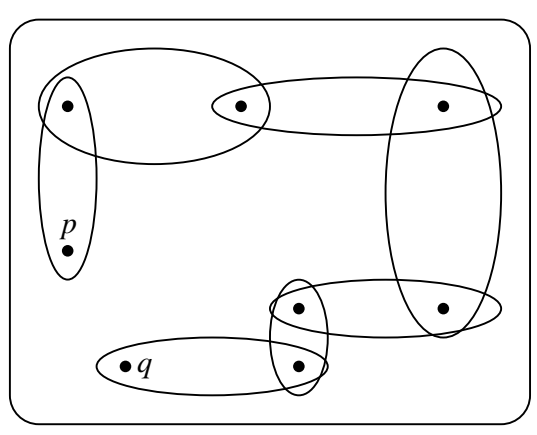

Fig. 2 A block (shown as a rounded rectangle) of the supremum of a family of partial partition is obtained by chaining blocks (shown as ellipses) of these partitions.

Then $(\Pi(E), \leq)$ is a complete lattice whose non-empty supremum and infimum operations are inherited from $\Pi^{*}(E)$; the least and greatest elements of $\Pi(E)$ are $\mathbf{0}_{E}$ and $\mathbf{1}_{E}$. For $A \in \mathcal{P}(E)$, the non-empty supremum and infimum operations in $\Pi(A)$ are inherited from $\Pi^{*}(A)$, hence from $\Pi^{*}(E)$; in other words a non-void supremum or infimum of partitions of $A$ is the same in $\Pi(A)$, in $\Pi^{*}(A)$ or in $\Pi^{*}(E)$.

Given a non-empty family $\left\{\pi_{i} \mid i \in I\right\}$ of partial partitions of $E$ such that for every $p \in E$, the set $\left\{\mathrm{Cl}_{\pi_{i}}(p) \mid i \in I\right\}$ is directed, we have [30,31]:

$$
\begin{gathered}
\forall p \in E, \quad \mathrm{Cl}_{\bigvee_{i \in I} \pi_{i}}(p)=\bigcup_{i \in I} \mathrm{Cl}_{\pi_{i}}(p) ; \\
\forall \pi \in \Pi^{*}(E), \quad \pi \wedge\left(\bigvee_{i \in I} \pi_{i}\right)=\bigvee_{i \in I}\left(\pi \wedge \pi_{i}\right) .
\end{gathered}
$$

Two conditions guaranteeing that $\left\{\mathrm{Cl}_{\pi_{i}}(p) \mid i \in I\right\}$ is directed for every $p \in E$, are: (A) the set $\left\{\pi_{i} \mid i \in I\right\}$ is directed; (B) for any two distinct $i, j \in I$, every non-singleton block of $\pi_{i}$ is disjoint from every non-singleton block of $\pi_{j}$.

An example of (4) for condition B is that for $B \in \mathcal{P}(E)$ and $\pi \in \Pi^{*}(E)$, we have $\pi \vee \mathbf{0}_{B}=\pi \cup \mathbf{0}_{B \backslash \operatorname{supp}(\pi)}$. One of (5) for condition $\mathrm{B}$ is that

$$
\forall \pi, \pi^{\prime} \in \Pi^{*}(E), \quad \pi \wedge \pi^{\prime}=\bigvee_{B \in \pi^{\prime}}\left(\pi \wedge \mathbf{1}_{B}\right)=\bigcup_{B \in \pi^{\prime}}\left(\pi \wedge \mathbf{1}_{B}\right)
$$

The support map supp : $\Pi^{*}(E) \rightarrow \mathcal{P}(E): \pi \mapsto \operatorname{supp}(\pi)$ is a complete morphism. Its upper adjoint is the erosion

$$
\text { 1. }: \mathcal{P}(E) \rightarrow \Pi^{*}(E): A \mapsto \mathbf{1}_{A},
$$

while its lower adjoint is the dilation

$$
\text { 0. : } \mathcal{P}(E) \rightarrow \Pi^{*}(E): A \mapsto \mathbf{0}_{A},
$$

in other words, for $\pi \in \Pi^{*}(E)$ and $A \in \mathcal{P}(E)$, $\operatorname{supp}(\pi) \subseteq A \Leftrightarrow \pi \leq \mathbf{1}_{A}$ and $A \subseteq \operatorname{supp}(\pi) \Leftrightarrow \mathbf{0}_{A} \leq \pi$. Since $\mathbf{1}_{\bullet}$ is an erosion and $\mathbf{0}_{\bullet}$ is a dilation, we have:

$$
\forall \mathcal{B} \subseteq \mathcal{P}(E), \quad \mathbf{0}_{\bigcup_{\mathcal{B}}}=\bigvee_{B \in \mathcal{B}} \mathbf{0}_{B} \quad \text { and } \quad \mathbf{1}_{\bigcap^{\mathcal{B}}}=\bigwedge_{B \in \mathcal{B}} \mathbf{1}_{B}
$$


Furthermore, the map supp is surjective, while the maps $\mathbf{1}_{\bullet}$ and $\mathbf{0}_{\bullet}$ are injective. Thus 1. and $\mathbf{0}$. are order-embeddings of the poset $\mathcal{P}(E)$ into the poset $\Pi^{*}(E)$ : for $A, B \in \mathcal{P}(E), A \subseteq B \Leftrightarrow \mathbf{0}_{A} \leq \mathbf{0}_{B} \Leftrightarrow \mathbf{1}_{A} \leq \mathbf{1}_{B}$.

\subsection{Partial connections}

We recall from [30] the definition and basic properties of partial connections, and of the complete lattice that they make. Unless we mention another source, all results and examples come from [30]. At the end of this subsection, Corollary 3 is a new result.

Write $\mathcal{S}(E)$ for the family of all singletons in $E: \mathcal{S}(E)=\{\{p\} \mid p \in E\}$. Formally, $\mathcal{S}(E)$ is the same set as $\mathbf{0}_{E}$, however we will use the notation $\mathbf{0}_{E}$ in the case of (partial) partitions, and $\mathcal{S}(E)$ in relation to (partial) connections.

A partial connection on $\mathcal{P}(E)$ is a family $\mathcal{C} \subseteq \mathcal{P}(E)$ such that $\emptyset \in \mathcal{C}$, and for any $\mathcal{B} \subseteq \mathcal{C}$ such that $\bigcap \mathcal{B} \neq \emptyset$, we have $\cup \mathcal{B} \in \mathcal{C}$. The partial connection $\mathcal{C}$ is a connection on $\mathcal{P}(E)$ if for all $p \in E,\{p\} \in \mathcal{C}$ (some authors $[29,17$, 5] call it a connectivity class). Note that $\mathcal{C} \subseteq \mathcal{P}(E)$ is a partial connection iff $\mathcal{C} \cup \mathcal{S}(E)$ is a connection; then $\mathcal{C} \cup \mathcal{S}(E)$ is the least connection containing $\mathcal{C}$. The elements of $\mathcal{C}$ are said to be $\mathcal{C}$-connected, and when there is no ambiguity about the underlying partial connection $\mathcal{C}$, we will call them connected.

The notion of connection unifies previous definitions of connectivity. For example, in a topological space, the family of connected sets (i.e., that cannot be partitioned by two open sets), and the family of arc-connected sets are two connections; the family of open connected sets and the one of open arcconnected sets are partial connections (in a metric space, the two coincide). In a graph, path-connected sets (i.e., sets of vertices where any two vertices are connected by a path), form a connection. Many other examples of (partial) connections have been given in $[36,29,17,5,30]$, we will will describe some of them later.

Suppose that to every point $p \in E$ is associated an opening $\gamma_{p}$ on $\mathcal{P}(E)$, and consider then the following properties that they may satisfy:

(C0a) For any $p \in E, \gamma_{p}(\{p\})=\{p\}$.

(C0b) For any $p \in E$ and $X \in \mathcal{P}(E), p \in X \Rightarrow p \in \gamma_{p}(X)$.

(C1a) For any $p \in E$ and $X \in \mathcal{P}(E), p \in X$ or $\gamma_{p}(X)=\emptyset$.

(C1b) For any $p \in E$ and $X \in \mathcal{P}(E), p \in \gamma_{p}(X)$ or $\gamma_{p}(X)=\emptyset$.

(C2a) For any $p, q \in E$ and $X \in \mathcal{P}(E), q \in \gamma_{p}(X) \Rightarrow \gamma_{p}(X)=\gamma_{q}(X)$.

Then $(\mathrm{C} 0 \mathrm{a})$ is equivalent to $(\mathrm{C} 0 \mathrm{~b})$, and $(\mathrm{C} 1 \mathrm{a})$ is equivalent to $(\mathrm{C} 1 \mathrm{~b})$.

A system of partial connection openings on $\mathcal{P}(E),\left(\gamma_{p}, p \in E\right)$, associates to each $p \in E$ an opening $\gamma_{p}$ on $\mathcal{P}(E)$, such that (C1a) (equivalently, (C1b)) and $(\mathrm{C} 2 \mathrm{a})$ are satisfied; each $\gamma_{p}$ is called the partial connection opening at $p$. If the $\gamma_{p}$ satisfy also (C0a) (equivalently, $\left.(\mathrm{C} 0 \mathrm{~b})\right)$, then $\left(\gamma_{p}, p \in E\right)$ is called a system of connection openings on $\mathcal{P}(E)$, and the $\gamma_{p}$ are called connection openings. Note that in a system of connection openings (i.e., when $(\mathrm{C} 0 \mathrm{a}) /(\mathrm{COb})$ holds), (C2a) can be replaced by: 
(C2b) For any $p, q \in E$ and $X \in \mathcal{P}(E), \gamma_{p}(X) \cap \gamma_{q}(X) \neq \emptyset \Rightarrow \gamma_{p}(X)=\gamma_{q}(X)$.

There exists a one-to-one correspondence between partial connections on $\mathcal{P}(E)$ and systems of partial connection openings on $\mathcal{P}(E)$. To every partial connection $\mathcal{C}$ corresponds the system of partial connection openings $\left(\gamma_{p}, p \in E\right)$ given by

$$
\forall p \in E, \forall X \in \mathcal{P}(E), \quad \gamma_{p}(X)=\bigcup\{C \in \mathcal{C} \mid p \in C, C \subseteq X\},
$$

and in fact: either the set $\{C \in \mathcal{C} \mid p \in C, C \subseteq X\}$ is empty and $\gamma_{p}(X)=\emptyset$, or $\gamma_{p}(X)$ is the greatest element of that set $\{C \in \mathcal{C} \mid p \in C, C \subseteq X\}$. Conversely, to every system of partial connection openings $\left(\gamma_{p}, p \in E\right)$ corresponds the partial connection

$$
\mathcal{C}=\left\{\gamma_{p}(X) \mid p \in E, X \in \mathcal{P}(E)\right\}
$$

Furthermore, $\mathcal{C}$ is a connection iff the corresponding $\left(\gamma_{p}, p \in E\right)$ is a system of connection openings on $\mathcal{P}(E)$.

Let $\mathcal{C}$ be a partial connection on $\mathcal{P}(E)$. For any $X \in \mathcal{P}(E)$, call a $\mathcal{C}$ component of $X$, or connected component of $X$ according to $\mathcal{C}$, any $C \in \mathcal{C}$ with $C \neq \emptyset$ and $C \subseteq X$, which is maximal for inclusion: $\forall C^{\prime} \in \mathcal{C}, C \subseteq C^{\prime} \subseteq$ $X \Rightarrow C^{\prime}=C$. This corresponds to the usual notion of connected component in topology.

Proposition 1 Let $\mathcal{C}$ be a partial connection on $\mathcal{P}(E)$. For any $X \in \mathcal{P}(E)$, the $\mathcal{C}$-components of $X$ are the $\gamma_{p}(X), p \in X$, for which $\gamma_{p}(X) \neq \emptyset$. They constitute a partial partition $\mathrm{PC}^{\mathcal{C}}(X)$, whose partial partition class map is $X \rightarrow \mathcal{P}(X): p \mapsto \gamma_{p}(X)$. When $\mathcal{C}$ is a connection, $\mathrm{PC}^{\mathcal{C}}(X)$ is a partition of $X$. The partial partition $\mathrm{PC}^{\mathcal{C}}(X)$ is isotone in $X: X \subseteq Y \Rightarrow \mathrm{PC}^{\mathcal{C}}(X) \leq \operatorname{PC}^{\mathcal{C}}(Y)$.

Note that $\mathrm{PC}^{\mathcal{C}}(X)$ is the finest partial partition $\pi$ of $X$ such that every $C \in \mathcal{C} \cap \mathcal{P}(X)$ is included in a block of $\pi$, and it is also the coarsest partial partition of $X$ whose blocks belong to $\mathcal{C}$.

Let us now give some examples of partial connections. Take $E=\mathbf{R}^{n}$ or $\mathbf{Z}^{n}$, provided with a standard connection $\mathcal{C}_{\text {std }}$ (the topological or arc connectivity for $\mathbf{R}^{n}$, the digital connectivity based on the 4 or 8 -adjacency on $\mathbf{Z}^{2}$, the 6,18 or 26 -adjacency on $\mathbf{Z}^{3}$, etc.). Choose a non-void $B \in \mathcal{C}_{\text {std }}$. Then the set $\mathcal{C}_{B}^{*}$ of all $Z \in \mathcal{C}_{\text {std }}$ such that $Z \circ B=Z$ (i.e., all connected unions of translates of $B$ ) forms a partial connection; for $X \notin \mathcal{C}_{B}^{*}$, the $\mathcal{C}_{B}^{*}$-components of $X$ are the $\mathcal{C}_{s t d}$-components of $X \circ B$, see Figure 3. The partial connection $\mathcal{C}_{B}^{*}$ allows to decompose a set $X$ into its "wide" parts, eliminating "narrow" portions and sharp corners. In fact, one has usually considered the connection $\mathcal{C}_{B}=\mathcal{C}_{B}^{*} \cup \mathcal{S}(E)[29]$.

Next, take $B \in \mathcal{C}_{s t d}$ containing the origin. The dilation by $B$ is extensive and it preserves $\mathcal{C}_{\text {std }}$ : for $X \in \mathcal{C}_{\text {std }}, X \subseteq X \oplus B$ and $X \oplus B \in \mathcal{C}_{\text {std }}$. Then the set $\mathcal{C}^{B}$ of all $X \in \mathcal{P}(E)$ such that $X \oplus B \in \mathcal{C}_{s t d}$ is a connection containing $\mathcal{C}_{\text {std }}[36]$. For $X \notin \mathcal{C}^{B}$, the $\mathcal{C}^{B}$-components of $X$ are obtained by intersecting 


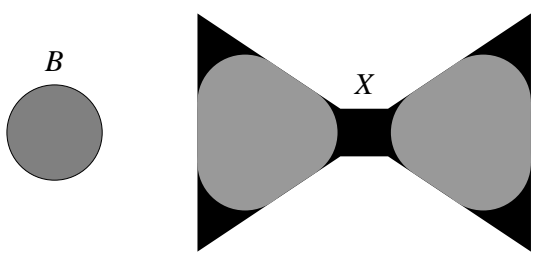

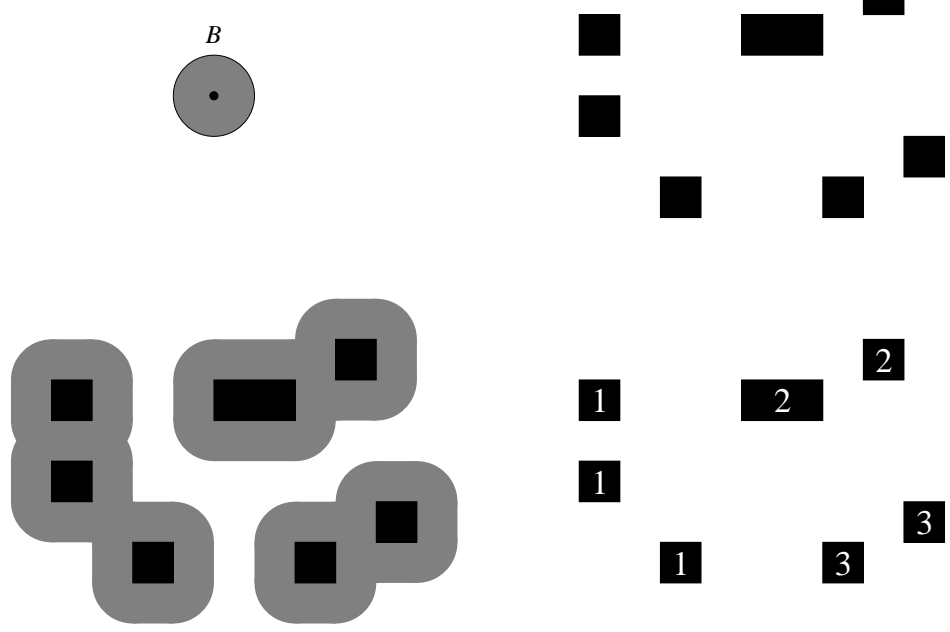

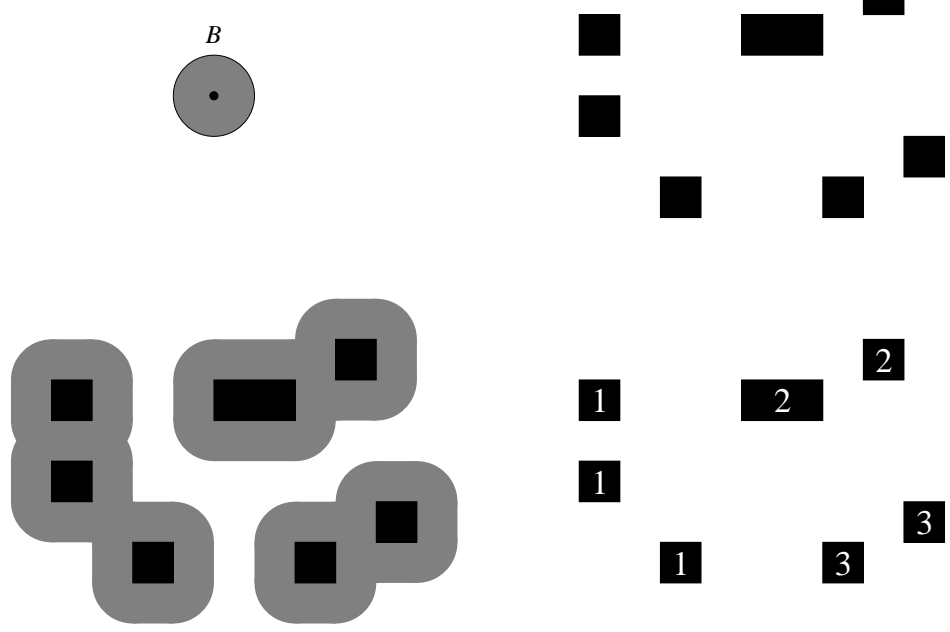

Fig. 3 Left: the structuring element $B \in \mathcal{C}_{\text {std }}$ is a disk. Right: the $\mathcal{C}_{B}^{*}$-components of the bowtie $X$ are the two $\mathcal{C}_{s t d^{-c o m p o n e n t s ~ o f ~ i t s ~}}$ opening $X \circ B$ (shown in grey). The points of the residual $X \backslash(X \circ B)$ (shown in black) do not belong to any $\mathcal{C}_{B}^{*}$-component, but they constitute singleton $\mathcal{C}_{B}$-components.

Fig. 4 Top left: the structuring element $B \in \mathcal{C}_{s t d}$ is a disk centered about the origin (shown as a black dot). Top right: the set $X$. Bottom left: the dilate $X \oplus B$ (in grey, with $X$ in black) has three connected components. Bottom right: the trace on $X$ of the three connected components of $X \oplus B$ are the three $\mathcal{C}^{B}$-components of $X$.

with $X$ the $\mathcal{C}_{\text {std }}$-components of $X \oplus B$, see Figure 4 . This connection allows to cluster neighbouring connected components of a set.

The above examples retain some similarity with the usual notion of connectivity, but there are stranger instances of partial connections, for example [29]: the family $\mathcal{C}_{N L S}^{n}$ of all subsets of $\mathbf{R}^{n}$ that are not linearly separable (i.e., such that there is no hyperplane disjoint from the set that separates it into two nonvoid parts), is a connection comprising all topologically connected sets. We show in Figure 5 a "pie" cut into three connected parts, such that the union of any two parts is "disconnected", but the union of all three is again "connected": this is incompatible with a connectivity arising from a topology or a graph adjacency.

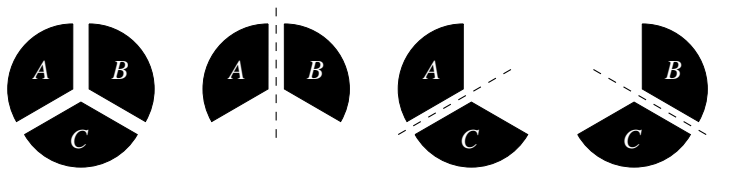

Fig. 5 In the connection $\mathcal{C}_{N L S}^{2}$ of all non linearly separable subsets of $\mathbf{R}^{2}$, we have $A, B, C \in$ $\mathcal{C}_{N L S}^{2}$, then $A \cup B, B \cup C, A \cup C \notin$ $\mathcal{C}_{N L S}^{2}$ but $A \cup B \cup C \in \mathcal{C}_{N L S}^{2}$. 
Dual Moore families are also partial connections; more precisely, a subset $\mathcal{M}$ of $\mathcal{P}(E)$ is a dual Moore family of $\mathcal{P}(E)$ iff it is a partial connection such that every set has at most one $\mathcal{M}$-component; given the opening $\gamma$ such that $\operatorname{lnv}(\gamma)=\mathcal{M}$, for every $X \in \mathcal{P}(E)$, the unique $\mathcal{M}$-component of $X$ is $\gamma(X)$ if $\gamma(X) \neq \emptyset$, while there is none if $\gamma(X)=\emptyset$. For example in Figure 3, taking for $\gamma$ the opening by $B$, the unique $\mathcal{M}$-component is the whole grey subset of $X$.

A particular case is when $\mathcal{M}=\{\emptyset\} \cup \mathcal{U}$, where $\mathcal{U}$ is an upper set. Thus the partial connection contains, besides the empty set, all subsets of $E$ that are "large enough". For example:

- For a discrete space $E$, given an integer $n>1$, all subsets of $E$ of size $\geq n$.

- For a fixed $A \in \mathcal{P}(E)$, all subsets of $E$ containing $A$.

- Given an isotone operator $\psi$ on $\mathcal{P}(E)$, all $X \in \mathcal{P}(E)$ such that $\psi(X) \neq \emptyset$; for instance, if $E=\mathbf{R}^{n}$ or $\mathbf{Z}^{n}$ and $\psi$ is the erosion by $B, \psi(X)=X \ominus B$, this gives all sets wide enough to contain a translate of $B$.

- Given a metric on $E$, all subsets of $E$ whose diameter exceeds some fixed value.

These families are not very interesting taken alone; however they can be useful if we take the intersection of the Moore family with the standard connection $\mathcal{C}_{\text {std }}$, for example: all connected sets whose diameter exceeds a given threshold.

Indeed, such an intersection gives a partial connection. More generally, any intersection of connections on $\mathcal{P}(E)$ is a connection on $\mathcal{P}(E)$, and any intersection of partial connections on $\mathcal{P}(E)$ is a partial connection on $\mathcal{P}(E)$, including the empty intersection $\mathcal{P}(E)$ that is a connection, hence a partial connection.

Thus intersection is a very useful way for generating new partial connections from existing ones. For example in Figure 1 we obtained a new segmentation by an infimum of connective criteria, i.e., by an intersection of connections $\mathcal{C}_{\mathrm{cr}}^{F}$. Also, the partial connection of Figure 3 is the intersection of the standard connection $\mathcal{C}_{\text {std }}$ and of the dual Moore family consisting of all $Z \in \mathcal{P}(E)$ such that $Z \circ B=Z$. Other examples can be given, for example the partial connection made of all $Z \in \mathcal{C}_{\text {std }}$ such that $Z \ominus B \neq \emptyset$, in other words such that $Z$ contains at least one translate of $B$.

Now given a family $\mathcal{B}$ of subsets of $E$, we define $\operatorname{Con}(\mathcal{B})$, the connection generated by $\mathcal{B}$, as the least connection containing $\mathcal{B}$; we define similarly $\operatorname{Con}^{*}(\mathcal{B})$, the partial connection generated by $\mathcal{B}$, as the least partial connection containing $\mathcal{B}$. Obviously, $\operatorname{Con}(\mathcal{B})=\operatorname{Con}\left(\operatorname{Con}^{*}(\mathcal{B})\right)=\operatorname{Con}^{*}(\mathcal{B}) \cup \mathcal{S}(E)$.

Let $\Gamma(E)$ be the set of all connections on $\mathcal{P}(E)$, and $\Gamma^{*}(E)$ the set of all partial connections on $\mathcal{P}(E)$. Then $\Gamma(E)$ and $\Gamma^{*}(E)$, ordered by inclusion, are atomistic complete lattices. In both the infimum operation is given by the intersection, including the empty intersection, or greatest element $\mathcal{P}(E)$. The supremum operation is given, in $\Gamma(E)$, by the connection generated by the union, while in $\Gamma^{*}(E)$, it is the partial connection generated by the union.

The intersection of partial connections can also be described in terms of systems of partial connection openings. Given two partial connections $\mathcal{C}$ and $\mathcal{C}^{\prime}$ with systems of partial connection openings $\left(\gamma_{p}, p \in E\right)$ and $\left(\gamma_{p}^{\prime}, p \in E\right)$, then 
$\mathcal{C} \subseteq \mathcal{C}^{\prime}$ iff for every $p \in E$ we have $\gamma_{p} \leq \gamma_{p}^{\prime}$. Given a non-void family $\mathcal{C}_{i}(i \in I$, $I \neq \emptyset)$ of partial connections with systems of partial connection openings $\left(\gamma_{p}^{i}, p \in E\right)$, then $\bigcap_{i \in I} \mathcal{C}_{i}$ has the system of partial connection openings $\left(\gamma_{p}, p \in\right.$ $E)$ such that for every $p \in E, \gamma_{p}$ is the greatest opening on $\mathcal{P}(E)$ that is $\leq \bigwedge_{i \in I} \gamma_{p}^{i}$, in other words, $\gamma_{p}$ is the infimum, in the lattice of openings on $\mathcal{P}(E)$, of the $\gamma_{p}^{i}, i \in I$.

A supremum of partial connections has no straightforward expression in terms of partial connection openings. However we have a nice result concerning the partition into connected components:

Proposition 2 For any $A \in \mathcal{P}(E)$, the map

$$
\Gamma^{*}(E) \rightarrow \Pi^{*}(A): \mathcal{C} \mapsto \mathrm{PC}^{\mathcal{C}}(A)
$$

is a dilation. The restriction to $\Gamma(E)$ of the map $\mathcal{C} \mapsto \mathrm{PC}^{\mathcal{C}}(A)$ is a dilation $\Gamma(E) \rightarrow \Pi(A)$.

This means that given a family $\mathcal{C}_{i}(i \in I)$ of partial connections, and $\mathcal{C}=\operatorname{Con}^{*}\left(\bigcup_{i \in I} \mathcal{C}_{i}\right), \mathrm{PC}^{\mathcal{C}}(A)=\bigvee_{i \in I} \mathrm{PC}^{\mathcal{C}_{i}}(A)$; in particular for $I$ empty, we have $\mathrm{PC}^{\{\emptyset\}}(A)=\varnothing$.

Note that [21] showed that the map associating to every topological space the partition of its connected components, preserves the coarse-to-fine ordering: a finer (i.e., greater) topology leads to a finer (i.e., smaller) partition into connected components.

A consequence of this proposition is that given a non-void family $\mathcal{B}$ of nonvoid subsets of $E, \operatorname{Con}^{*}(\mathcal{B})$ is the set of all $X \in \mathcal{P}(E)$ that are chained by $\mathcal{B} \cap$ $\mathcal{P}(X)$. In other words, the construction of a supremum of partial connections uses the same chaining operation as in a supremum of partial partitions. This "coincidence" had been remarked previously in the case of connections and partitions $[29,39]$.

In [12] a "connectivity system" is built from a family $\mathcal{B} \subseteq \mathcal{P}(E)$; it consists in all subsets $X$ of $E$ such that any two distinct points of $X$ are chained by blocks in $\mathcal{B} \cap \mathcal{P}(X)$, in other words it is $\operatorname{Con}(\mathcal{B})$, the connection generated by $\mathcal{B}$.

Note that there is no analogue of Proposition 2 for the infimum operation. Combining Proposition 2 with the fact that $\mathrm{Cl}_{\mathrm{PC}^{\mathcal{C}}(A)}(p)=\gamma_{p}(A)$ (see Proposition 1), and with (4) in condition A below it, we get:

Corollary 3 Given a directed family $\mathcal{C}_{i}(i \in I)$ of partial connections with systems of partial connection openings $\left(\gamma_{p}^{i}, p \in E\right)$, and their supremum $\mathcal{C}=$ Con $^{*}\left(\bigcup_{i \in I} \mathcal{C}_{i}\right)$ with system of partial connection openings $\left(\gamma_{p}, p \in E\right)$, then for any $p \in E$ and $A \in \mathcal{P}(E)$ we have $\gamma_{p}(A)=\bigcup_{i \in I} \gamma_{p}^{i}(A)$.

\section{Block splitting operators and openings}

We consider anti-extensive operators on partial partitions, that operate by splitting each block independently. We are particularly interested in the design 
of idempotent operators. From a practical point of view, such a block splitting operator is often derived from an image $F: E \rightarrow T$ and a criterion $\mathrm{cr}$, then it is applied to the universal partition $\mathbf{1}_{E}$ to produce the partial partition representing the segmentation of the image $F$ on $E$.

Subsection 3.1 studies the relations between a set splitting operator and the resulting block splitting operator on partial partitions. Subsection 3.2 characterizes block splitting openings, they coincide with operators splitting each block into its $\mathcal{C}$-components for a partial connection $\mathcal{C}$; this result is related to another characterization of connections and partial connections [39,30]. These operators underlie Serra's approach to segmentation [32,39].

For any complete lattice $L$, write $A E(L)$ for the set of anti-extensive operators on $L$. A general reminder: $A E(L)$ is a complete lattice, it has the same non-void supremum and infimum operations as the power lattice of all operators $L \rightarrow L$; both lattices have also the same empty supremum, or least element, namely the constant operator $x \mapsto \mathbf{0}$. However the empty infimum, or greatest element, is the identity operator id : $x \mapsto x$ in $A E(L)$, while in the lattice of all operators, it is the constant operator $x \mapsto \mathbf{1}$. Write $A E^{I}(L)$ for the set of isotone anti-extensive operators on $L$; it is a complete sublattice of $A E(L)$.

\subsection{Set splitting and block splitting}

Let us start with a few technical details about the family of partial partitions that are below a given partial partition. Let us fix $\pi^{*} \in \Pi^{*}(E)$. For any $\pi \in \Pi^{*}(E)$ such that $\pi \leq \pi^{*}$, the blocks of $\pi^{*}$ induce a partition of $\pi$; for any $B \in \pi^{*}$, the restriction of $\pi$ to $B$ is the set $[\pi]_{B}$ of blocks of $\pi$ that are included in $B$ :

$$
\pi \leq \pi^{*}, B \in \pi^{*}: \quad[\pi]_{B}=\pi \cap \mathcal{P}(B)=\pi \wedge \mathbf{1}_{B}
$$

By extension, we set $[\pi]_{\emptyset}=\varnothing$. In term of point classes, we have:

$$
\pi \leq \pi^{*}, B \in \pi^{*}, p \in E: \quad \mathrm{Cl}_{[\pi]_{B}}(p)= \begin{cases}\mathrm{Cl}_{\pi}(p) & \text { if } p \in B \\ \emptyset & \text { if } p \notin B\end{cases}
$$

Then the $[\pi]_{B}, B \in \pi^{*}$, are pairwise disjoint, more precisely they have pairwise disjoint supports, and their union gives $\pi$ :

$$
\pi \leq \pi^{*}: \quad \pi=\bigcup_{B \in \pi^{*}}[\pi]_{B}=\bigvee_{B \in \pi^{*}}[\pi]_{B}
$$

The restriction to a block $B$ is compatible with refinement order:

$$
\pi, \pi^{\prime} \leq \pi^{*}: \quad \pi \leq \pi^{\prime} \quad \Longleftrightarrow \quad\left(\forall B \in \pi^{*},[\pi]_{B} \leq\left[\pi^{\prime}\right]_{B}\right)
$$


In particular, $\pi=\pi^{\prime}$ iff for all $B \in \pi^{*},[\pi]_{B}=\left[\pi^{\prime}\right]_{B}$. Given a non-void family of partial partitions below $\pi^{*}$, the infimum and supremum are compatible with the restriction to a block $B$ :

$$
\begin{aligned}
& \pi_{i} \leq \pi^{*}, i \in I \neq \emptyset, B \in \pi^{*}: \\
& {\left[\bigwedge_{i \in I} \pi_{i}\right]_{B}=\bigwedge_{i \in I}\left[\pi_{i}\right]_{B} \text { and }\left[\bigvee_{i \in I} \pi_{i}\right]_{B}=\bigvee_{i \in I}\left[\pi_{i}\right]_{B} .}
\end{aligned}
$$

Indeed, if we have a non-void intersection of blocks, one from each $\pi_{i}$, or a chaining of blocks from the union of all $\pi_{i}$ 's, then these blocks must all be included in the same block of $\pi^{*}$. Alternately, this can be deduced from $(10,11)$. We illustrate $(11,12)$ in Figure 6.
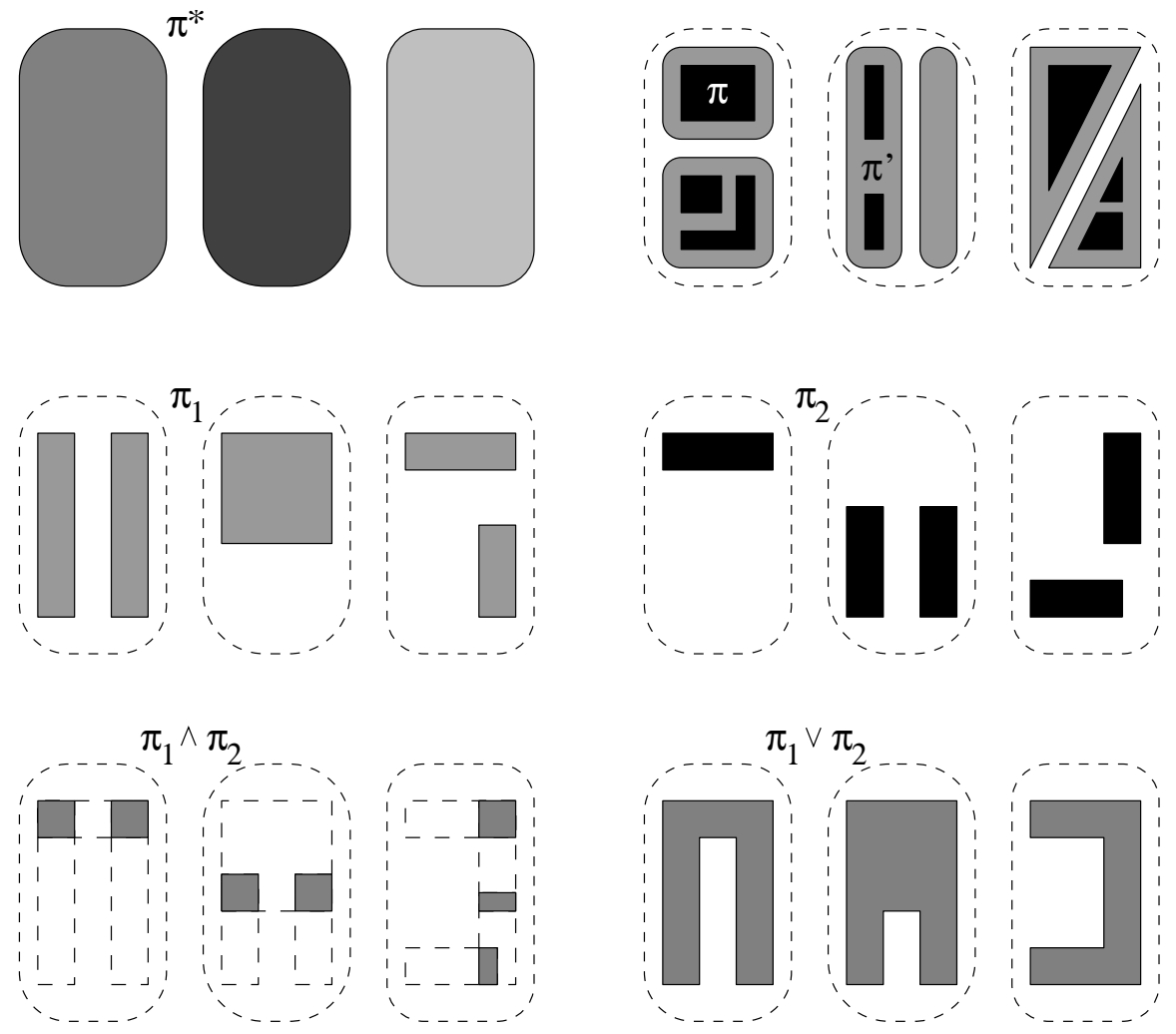

Fig. 6 Top left: the partial partition $\pi^{*}$. Top right: for $\pi, \pi^{\prime} \leq \pi^{*}$, checking that each block of $\pi$ (in black) is included in a block of $\pi^{\prime}$ (in grey) can be done by such a check inside every block of $\pi^{*}$, thus (11) holds. Middle row: $\pi_{1}, \pi_{2} \leq \pi^{*}$. Bottom left: the intersection of blocks of $\pi_{1}$ with those of $\pi_{2}$ always happens inside a block of $\pi^{*}$. Bottom right: the chaining of blocks of $\pi_{1}$ and $\pi_{2}$ can be done only inside a block of $\pi^{*}$. Hence (12) holds. 
Let us now define set splitting operators and the corresponding block splitting operators on partial partitions; the latter will also be characterized in Corollary 7.

Definition 4 A set splitting operator on $\mathcal{P}(E)$ is a map $\sigma: \mathcal{P}(E) \rightarrow \Pi^{*}(E)$ such that for every $X \in \mathcal{P}(E), \sigma(X) \in \Pi^{*}(X)$. If for every $X \in \mathcal{P}(E)$, $\sigma(X) \in \Pi(X)$, then we say that $\sigma$ is support-preserving. The block splitting operator on $\Pi^{*}(E)$ derived from $\sigma$ is the map $\beta(\sigma): \Pi^{*}(E) \rightarrow \Pi^{*}(E)$ that applies $\sigma$ to each block of a partial partition, in other words

$$
\forall \pi \in \Pi^{*}(E), \quad \beta(\sigma)(\pi)=\bigcup_{B \in \pi} \sigma(B) ;
$$

equivalently,

$$
\forall \pi \in \Pi^{*}(E), \forall B \in \pi, \quad[\beta(\sigma)(\pi)]_{B}=\sigma(B) .
$$

Write $\Sigma(E)$ for the set of all set splitting operators on $\mathcal{P}(E)$.

Note that we always have $\sigma(\emptyset)=\varnothing$, and a block on which $\sigma$ is applied, is always non-void. Thus a set splitting operator needs only to be defined on $\mathcal{P}(E) \backslash\{\emptyset\}$. When $\sigma$ is support-preserving, then $\beta(\sigma)$ is also support-preserving: for all $\pi \in \Pi^{*}(E), \operatorname{supp}(\beta(\sigma)(\pi))=\operatorname{supp}(\pi)$; in particular, if $\pi \in \Pi(E)$, then $\beta(\sigma)(\pi) \in \Pi(E)$. Conversely, if for some $X \in \mathcal{P}(E) \backslash\{\emptyset\}$ we have $\sigma(X) \notin$ $\Pi(X)$, then taking any partition $\pi$ with $X \in \pi$, we get $\beta(\sigma)(\pi) \notin \Pi(E)$.

Proposition 5 For any set splitting operator $\sigma$ on $\mathcal{P}(E)$ :

1. for any $A \in \mathcal{P}(E), \beta(\sigma)\left(\mathbf{1}_{A}\right)=\sigma(A)$;

2. for any $p \in E$ and $\pi \in \Pi^{*}(E), \mathrm{Cl}_{\beta(\sigma)(\pi)}(p)=\mathrm{Cl}_{\sigma\left(\mathrm{Cl}_{\pi}(p)\right)}(p)$, see Figure 7;

3. $\beta(\sigma)$ is anti-extensive;

4. $\beta(\sigma)$ is isotone iff $\sigma$ is isotone.

$\Sigma(E)$ is a complete lattice, and $\beta$ is an injective complete morphism (i.e., a complete embedding) from $\Sigma(E)$ into $A E\left(\Pi^{*}(E)\right)$, the complete lattice of anti-extensive operators on $\Pi^{*}(E)$.

Proof 1. If $A=\emptyset$, then $\mathbf{1}_{A}=\emptyset$; now by definition $\sigma(\emptyset)=\emptyset$ and $\beta(\sigma)(\varnothing)=$ $\varnothing$. Suppose now that $A \neq \emptyset$, thus $\mathbf{1}_{A}=\{A\}$, and by definition we get $\beta(\sigma)(\{A\})=\sigma(A)$

3. For $B \in \mathcal{P}(E), \sigma(B) \in \Pi^{*}(B)$, so $\sigma(B) \leq \mathbf{1}_{B}$. Thus for $\pi \in \Pi^{*}(E)$,

$$
\beta(\sigma)(\pi)=\bigvee_{B \in \pi} \sigma(B) \leq \bigvee_{B \in \pi} \mathbf{1}_{B}=\pi
$$

2. See Figure 7. If $p \notin \operatorname{supp}(\pi)$, then $\mathrm{Cl}_{\pi}(p)=\emptyset$, so $\sigma\left(\mathrm{Cl}_{\pi}(p)\right)=\sigma(\emptyset)=$ $\emptyset$, hence $\mathrm{Cl}_{\sigma\left(\mathrm{Cl}_{\pi}(p)\right)}(p)=\mathrm{Cl}_{\emptyset}(p)=\emptyset$. As $\beta(\sigma)$ is anti-extensive (item 3), $\beta(\sigma)(\pi) \leq \pi$, so $p \notin \operatorname{supp}(\beta(\sigma)(\pi))$, and $\mathrm{Cl}_{\beta(\sigma)(\pi)}(p)=\emptyset$. Thus $\mathrm{Cl}_{\beta(\sigma)(\pi)}(p)=$ $\mathrm{Cl}_{\sigma\left(\mathrm{Cl}_{\pi}(p)\right)}(p)$. Suppose now that $p \in \operatorname{supp}(\pi)$, so $\mathrm{Cl}_{\pi}(p)=A \in \pi$. For any 


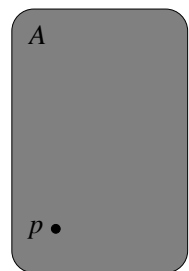

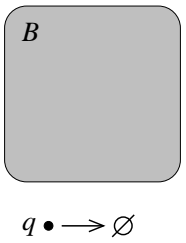

$\pi$

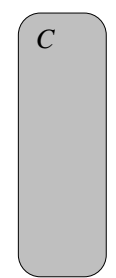

(1)
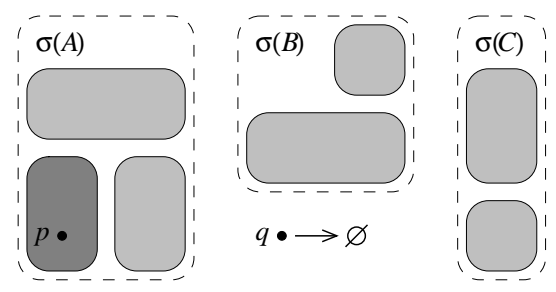

$\beta(\sigma)(\pi)$

Fig. 7 Illustration of item 2 of Proposition 5. Here $\mathrm{Cl}_{\pi}(p)=A$ and $\mathrm{Cl}_{\beta(\sigma)(\pi)}(p)=\mathrm{Cl}_{\sigma(A)}(p)$; on the other hand $q \notin \operatorname{supp}(\pi)$ and $\mathrm{Cl}_{\pi}(q)=\mathrm{Cl}_{\beta(\sigma)(\pi)}(q)=\emptyset$.

$B \in \pi \backslash\{A\}, p \notin B$, and as $\sigma(B) \in \Pi^{*}(B), p$ may not belong to a class of $\sigma(B)$. Thus in $\beta(\sigma)(\pi)=\bigcup_{B \in \pi} \sigma(B), p$ may belong only to a class of $\sigma(A)$, that is, $\mathrm{Cl}_{\beta(\sigma)(\pi)}(p)=\mathrm{Cl}_{\sigma(A)}(p)=\mathrm{Cl}_{\sigma\left(\mathrm{Cl}_{\pi}(p)\right)}(p)$.

4. Suppose that $\sigma$ is isotone, and let $\pi, \pi^{\prime} \in \Pi^{*}(E)$ such that $\pi \leq \pi^{\prime}$. Then every block $B$ of $\pi$ is included in some block $C$ of $\pi^{\prime}$; as $B \subseteq C$ and $\sigma$ is isotone, $\sigma(B) \leq \sigma(C)$, in other words every block of $\sigma(B)$ is included in some block of $\sigma(C)$; as $\beta(\sigma)(\pi)=\bigcup_{B \in \pi} \sigma(B)$ (and similarly for $\beta(\sigma)\left(\pi^{\prime}\right)$ ), every block of $\beta(\sigma)(\pi)$ is included in some block of $\beta(\sigma)\left(\pi^{\prime}\right)$, that is, $\beta(\sigma)(\pi) \leq \beta(\sigma)\left(\pi^{\prime}\right)$. Hence $\beta(\sigma)$ is isotone.

Conversely, suppose that $\beta(\sigma)$ is isotone, and let $A, B \in \mathcal{P}(E)$ such that $A \subseteq B$. Then $\mathbf{1}_{A} \leq \mathbf{1}_{B}$, and as $\beta(\sigma)$ is isotone, $\beta(\sigma)\left(\mathbf{1}_{A}\right) \leq \beta(\sigma)\left(\mathbf{1}_{B}\right)$. By item 1, this gives $\sigma(A) \leq \sigma(B)$. Hence $\sigma$ is isotone.

Let us now show the last sentence of the statement. In $\Pi^{*}(E)^{\mathcal{P}(E)}$, the power lattice of all maps $\mathcal{P}(E) \rightarrow \Pi^{*}(E), \Sigma(E)$ is the set of maps that are $\leq \mathbf{1}_{\bullet}: A \mapsto \mathbf{1}_{A}$. Thus it is a complete lattice with greatest element 1. By item 3, $\beta$ maps $\Sigma(E)$ into $A E\left(\Pi^{*}(E)\right)$, and by item $1, \beta$ is injective. Let $\pi \in \Pi^{*}(E)$ and consider a non-void family $\sigma_{i}, i \in I \neq \emptyset$, of set splitting operators. Combining (14) and (12), for any $B \in \pi$ we have

$$
\begin{gathered}
{\left[\beta\left(\bigvee_{i \in I} \sigma_{i}\right)(\pi)\right]_{B}=\left(\bigvee_{i \in I} \sigma_{i}\right)(B)=\bigvee_{i \in I} \sigma_{i}(B)} \\
=\bigvee_{i \in I}\left[\beta\left(\sigma_{i}\right)(\pi)\right]_{B}=\left[\bigvee_{i \in I} \beta\left(\sigma_{i}\right)(\pi)\right]_{B}=\left[\left(\bigvee_{i \in I} \beta\left(\sigma_{i}\right)\right)(\pi)\right]_{B},
\end{gathered}
$$

from which we deduce that $\beta\left(\bigvee_{i \in I} \sigma_{i}\right)(\pi)=\left(\bigvee_{i \in I} \beta\left(\sigma_{i}\right)\right)(\pi)$; thus $\beta\left(\bigvee_{i \in I} \sigma_{i}\right)$ $=\bigvee_{i \in I} \beta\left(\sigma_{i}\right)$. We obtain the same with $\bigwedge_{i \in I} \sigma_{i}$. Hence $\beta$ commutes with non-empty suprema and infima. Now in $\Sigma(E)$ the empty supremum, or least element, is the constant $\mathbf{0}_{\Sigma}: X \mapsto \varnothing$, with $\beta\left(\mathbf{0}_{\Sigma}\right): \pi \mapsto \bigcup_{B \in \pi} \varnothing=\varnothing$, which is the least anti-extensive operator on $\Pi^{*}(E)$. Finally, in $\Sigma(E)$ the empty infimum, or greatest element, is $\mathbf{1}_{\bullet}: X \mapsto \mathbf{1}_{X}$, which gives $\beta\left(\mathbf{1}_{\bullet}\right): \pi \mapsto$ $\bigcup_{B \in \pi} \mathbf{1}_{B}=\pi$, in other words the identity on $\Pi^{*}(E)$, which is the greatest antiextensive operator. Therefore $\beta$ is a complete morphism $\Sigma(E) \rightarrow A E\left(\Pi^{*}(E)\right)$. 
Since $\Sigma(E)$ is a complete lattice, and $\beta: \Sigma(E) \rightarrow A E\left(\Pi^{*}(E)\right)$ is a complete morphism, it follows that the family of block splitting operators is a complete sublattice of $A E\left(\Pi^{*}(E)\right)$. The following example will be used in Subsection 3.2:

Example 6 Take a fixed non-void $\pi_{0} \in \Pi^{*}(E)$, and let $\sigma$ be the set splitting operator given by $\sigma(B)=\mathbf{1}_{B} \wedge \pi_{0}$. The block splitting operator $\beta(\sigma)$ satisfies $\beta(\sigma)(\pi)=\pi \wedge \pi_{0}$ by $(6)$. Note that $\sigma$ is isotone and $\beta(\sigma)$ is an opening. When $\pi_{0} \in \Pi(E), \sigma$ is support-preserving.

Corollary 7 Let $\eta$ be an anti-extensive operator on $\Pi^{*}(E)$. Then $\eta$ is a block splitting operator iff for any $\pi \in \Pi^{*}(E), \eta(\pi)=\bigcup_{B \in \pi} \eta\left(\mathbf{1}_{B}\right)$.

Proof Given a set splitting operator $\sigma,(13)$ and item 1 of Proposition 5 give for any $\pi \in \Pi^{*}(E)$ :

$$
\beta(\sigma)(\pi)=\bigcup_{B \in \pi} \sigma(B)=\bigcup_{B \in \pi} \beta(\sigma)\left(\mathbf{1}_{B}\right),
$$

thus $\eta=\beta(\sigma)$ has the required property.

Conversely, let $\eta$ be an anti-extensive operator on $\Pi^{*}(E)$ such that for any $\pi \in \Pi^{*}(E), \eta(\pi)=\bigcup_{B \in \pi} \eta\left(\mathbf{1}_{B}\right)$. Define $\sigma$ by setting $\sigma(B)=\eta\left(\mathbf{1}_{B}\right)$ for any $B \in \mathcal{P}(E)$; as $\eta$ is anti-extensive, $\eta\left(\mathbf{1}_{B}\right) \leq \mathbf{1}_{B}$, that is, $\sigma(B) \in \Pi^{*}(B)$, and $\sigma$ is a set splitting operator. By (13) we get then for any $\pi \in \Pi^{*}(E)$ :

$$
\eta(\pi)=\bigcup_{B \in \pi} \eta\left(\mathbf{1}_{B}\right)=\bigcup_{B \in \pi} \sigma(B)=\beta(\sigma)(\pi)
$$

thus $\eta=\beta(\sigma)$.

Corollary 8 The family of block splitting operators is a monoid.

Proof The operator $\mathbf{1}_{\bullet}: X \mapsto \mathbf{1}_{X}$ is set splitting, and the derived block splitting operator $\beta\left(\mathbf{1}_{\bullet}\right)$ is the identity on $\Pi^{*}(E)$. Now let $\eta$ and $\zeta$ be block splitting operators. Let $\pi \in \Pi^{*}(E)$; we apply Corollary 7:

$$
\begin{aligned}
\zeta \eta(\pi) & =\bigcup\left\{\zeta\left(\mathbf{1}_{C}\right) \mid C \in \eta(\pi)\right\}=\bigcup\left\{\zeta\left(\mathbf{1}_{C}\right) \mid C \in \bigcup_{B \in \pi} \eta\left(\mathbf{1}_{B}\right)\right\} \\
& =\bigcup\left(\bigcup\left\{\zeta\left(\mathbf{1}_{C}\right) \mid C \in \eta\left(\mathbf{1}_{B}\right)\right\}\right)=\bigcup_{B \in \pi} \zeta\left(\eta\left(\mathbf{1}_{B}\right)\right)
\end{aligned}
$$

see Figure 8, so $\zeta \eta$ is block splitting.

The complete morphism $\beta: \Sigma(E) \rightarrow A E\left(\Pi^{*}(E)\right)$ has both an upper and a lower adjoint $A E\left(\Pi^{*}(E)\right) \rightarrow \Sigma(E)$. Define $\beta^{+}, \beta^{-}: A E\left(\Pi^{*}(E)\right) \rightarrow \Sigma(E)$ by setting $\forall \eta \in A E\left(\Pi^{*}(E)\right): \beta^{+}(\eta)(\emptyset)=\beta^{-}(\eta)(\emptyset)=\emptyset$, and $\forall B \in \mathcal{P}(E) \backslash\{\emptyset\}$ :

$$
\begin{aligned}
& \beta^{+}(\eta)(B)=\bigwedge_{\pi \in \Pi^{*}(E \backslash B)}\left[\eta\left(\mathbf{1}_{B} \cup \pi\right)\right]_{B}=\left[\bigwedge_{\pi \in \Pi^{*}(E \backslash B)} \eta\left(\mathbf{1}_{B} \cup \pi\right)\right]_{B}, \\
& \beta^{-}(\eta)(B)=\bigvee_{\pi \in \Pi^{*}(E \backslash B)}\left[\eta\left(\mathbf{1}_{B} \cup \pi\right)\right]_{B}=\left[\bigvee_{\pi \in \Pi^{*}(E \backslash B)} \eta\left(\mathbf{1}_{B} \cup \pi\right)\right]_{B} .
\end{aligned}
$$



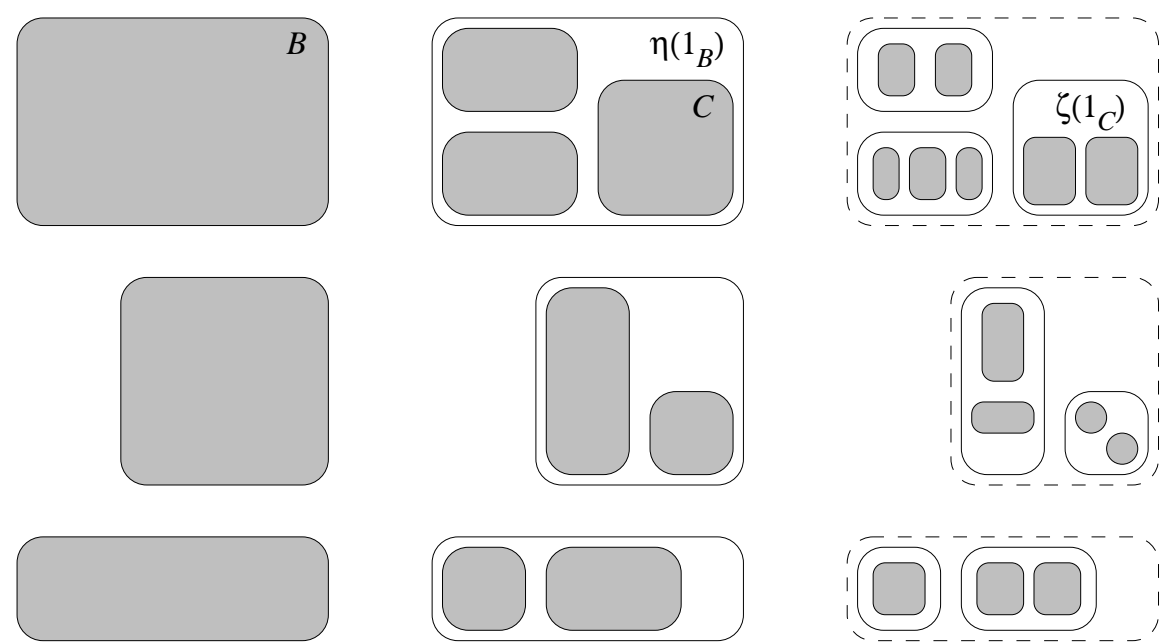

$\pi$
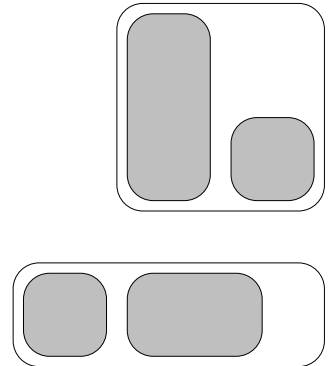

$\eta(\pi)$
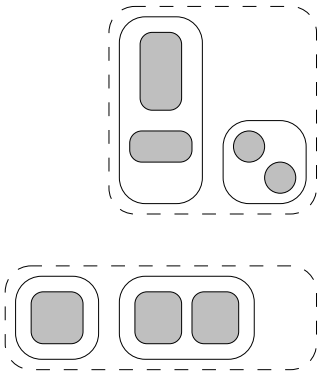

$\zeta(\eta(\pi))$

Fig. 8 Illustration of Corollary 8. Here $B \in \pi$ and $C \in \eta\left(\mathbf{1}_{B}\right)$.

The two rightmost equalities follow from (12). By (8), in the above, each of the four $[\ldots]_{B}$ can be replaced by $(\ldots) \wedge \mathbf{1}_{B}$.

Proposition 9 The two pairs $\left(\beta^{+}, \beta\right): A E\left(\Pi^{*}(E)\right) \rightleftharpoons \Sigma(E)$ and $\left(\beta, \beta^{-}\right)$: $\Sigma(E) \rightleftharpoons A E\left(\Pi^{*}(E)\right)$ are adjunctions, $\beta^{+}$and $\beta^{-}$are surjective and $\beta^{+} \beta=$ $\beta^{-} \beta$ is the identity on $\Sigma(E)$. For any anti-extensive operator $\eta$ on $\Pi^{*}(E)$, the following five statements are equivalent: (a) $\eta$ is a block splitting operator; (b) $\beta \beta^{+}(\eta)=\eta$; (c) $\beta \beta^{-}(\eta)=\eta$; (d) $\beta \beta^{+}(\eta)=\beta \beta^{-}(\eta)$; (e) $\beta^{+}(\eta)=\beta^{-}(\eta)$.

Proof Let $\sigma \in \Sigma(E)$ and $\eta \in A E\left(\Pi^{*}(E)\right)$. Since $\sigma(\emptyset)=\beta^{+}(\eta)(\emptyset)=\emptyset$, we have $\sigma \leq \beta^{+}(\eta)$ iff $\forall B \in \mathcal{P}(E) \backslash\{\emptyset\}, \sigma(B) \leq \beta^{+}(\eta)(B)=\bigwedge_{\pi \in \Pi^{*}(E \backslash B)}\left[\eta\left(\mathbf{1}_{B} \cup\right.\right.$ $\pi)]_{B}$, in other words iff $\forall B \in \mathcal{P}(E) \backslash\{\emptyset\}, \forall \pi \in \Pi^{*}(E \backslash B), \sigma(B) \leq\left[\eta\left(\mathbf{1}_{B} \cup\right.\right.$ $\pi)]_{B}$. Now the map $\pi \mapsto \mathbf{1}_{B} \cup \pi=\pi^{\prime}$ induces a one-to-one correspondence between pairs $\left(B \in \mathcal{P}(E) \backslash\{\emptyset\}, \pi \in \Pi^{*}(E \backslash B)\right)$ and pairs $\left(\pi^{\prime} \in \Pi^{*}(E), B \in\right.$ $\left.\pi^{\prime}\right)$. So the condition becomes $\forall \pi^{\prime} \in \Pi^{*}(E), \forall B \in \pi^{\prime}, \sigma(B) \leq\left[\eta\left(\pi^{\prime}\right)\right]_{B}$, which by (14) means $\left[\beta(\sigma)\left(\pi^{\prime}\right)\right]_{B} \leq\left[\eta\left(\pi^{\prime}\right)\right]_{B}$, and by (11) this is equivalent to $\forall \pi^{\prime} \in \Pi^{*}(E), \beta(\sigma)\left(\pi^{\prime}\right) \leq \eta\left(\pi^{\prime}\right)$, that is, $\beta(\sigma) \leq \eta$. Hence $\left(\beta^{+}, \beta\right)$ is an adjunction. The proof that $\left(\beta, \beta^{-}\right)$is an adjunction is dual by inverting the order $(\leq \leftrightarrow \geq, \bigwedge \leftrightarrow \bigvee)$.

The remaining statements follow from the two adjunctions $\left(\beta^{+}, \beta\right),\left(\beta, \beta^{-}\right)$ and the injectivity of $\beta$, using classical results $[4,7,13,16]$. In particular we easily show that $(\mathrm{a}) \Rightarrow(\mathrm{e}) \Rightarrow(\mathrm{d}) \Rightarrow(\mathrm{b}, \mathrm{c})$, then $(\mathrm{b}) \Rightarrow(\mathrm{a})$ and $(\mathrm{c}) \Rightarrow(\mathrm{a})$.

For any anti-extensive operator $\eta$ on $\Pi^{*}(E)$, define:

$$
\begin{aligned}
& \rho(\eta): \mathcal{P}(E) \rightarrow \Pi^{*}(E): B \mapsto \eta\left(\mathbf{1}_{B}\right) \\
& \theta(\eta): \mathcal{P}(E) \rightarrow \Pi^{*}(E): B \mapsto\left[\eta\left(\mathbf{1}_{B} \cup \mathbf{1}_{E \backslash B}\right)\right]_{B},
\end{aligned}
$$


with $\rho(\eta)(\emptyset)=\theta(\eta)(\emptyset)=\emptyset$. The remark made after (15) applies here, so

$$
\theta(\eta)(B)=\eta\left(\mathbf{1}_{B} \cup \mathbf{1}_{E \backslash B}\right) \wedge \mathbf{1}_{B} .
$$

Clearly $\rho(\eta)$ and $\theta(\eta)$ are set splitting operators.

Proposition 10 If $\eta$ is isotone, then $\beta^{+}(\eta)=\rho(\eta), \beta^{-}(\eta)=\theta(\eta)$ and $\rho(\eta)$ is isotone.

Proof We have $\beta^{+}(\eta)(\emptyset)=\rho(\eta)(\emptyset)=\beta^{-}(\eta)(\emptyset)=\theta(\eta)(\emptyset)=\varnothing$. Let $B \in$ $\mathcal{P}(E) \backslash\{\emptyset\}$. For any $\pi \in \Pi^{*}(E \backslash B)$ we have $\mathbf{1}_{B} \leq \mathbf{1}_{B} \cup \pi \leq \mathbf{1}_{B} \cup \mathbf{1}_{E \backslash B}$, so $\eta\left(\mathbf{1}_{B}\right) \leq \eta\left(\mathbf{1}_{B} \cup \pi\right) \leq \eta\left(\mathbf{1}_{B} \cup \mathbf{1}_{E \backslash B}\right)$, hence by (11) we get $\eta\left(\mathbf{1}_{B}\right)=\left[\eta\left(\mathbf{1}_{B}\right)\right]_{B} \leq$ $\left[\eta\left(\mathbf{1}_{B} \cup \pi\right)\right]_{B} \leq\left[\eta\left(\mathbf{1}_{B} \cup \mathbf{1}_{E \backslash B}\right)\right]_{B}$; thus $(15)$ gives $\beta^{+}(\eta)(B)=\rho(\eta)(B)$ and $\beta^{-}(\eta)(B)=\theta(\eta)(B)$. Now $\rho(\eta)$ is the composition of the two isotone maps $\eta$ and $\mathbf{1}_{\bullet}$, so it is isotone.

Note that $\theta(\eta)$ is usually not isotone (because it involves the antitone map $B \mapsto E \backslash B)$. Now let $\Sigma^{I}(E)$ be the set of isotone set splitting operators, and recall $A E^{I}\left(\Pi^{*}(E)\right)$ the set of isotone anti-extensive operators on $\Pi^{*}(E)$. Both are complete lattices, and by Proposition $5, \beta$ induces a complete morphism $\Sigma^{I}(E) \rightarrow A E^{I}\left(\Pi^{*}(E)\right)$; now $\rho$ restricted to $A E^{I}\left(\Pi^{*}(E)\right)$ coincides with the restriction of $\beta^{+}$to $A E^{I}\left(\Pi^{*}(E)\right)$, and is a map $A E^{I}\left(\Pi^{*}(E)\right) \rightarrow \Sigma^{I}(E)$. Thus if we restrict $\beta$ to $\Sigma^{I}(E)$ and $\rho$ to $A E^{I}\left(\Pi^{*}(E)\right)$, then $(\rho, \beta)$ is an adjunction $A E^{I}\left(\Pi^{*}(E)\right) \rightleftharpoons \Sigma^{I}(E)$. However $(\beta, \theta)$ is not an adjunction $\Sigma^{I}(E) \rightleftharpoons$ $A E^{I}\left(\Pi^{*}(E)\right)$, because $\theta$ is not $A E^{I}\left(\Pi^{*}(E)\right) \rightarrow \Sigma^{I}(E)$.

Let us illustrate $\rho(\eta)$ and $\theta(\eta)$ with two examples of an isotone $\eta$ :

1. Assume $E=\mathbf{R}^{n}$ or $E=\mathbf{Z}^{n}$, and let $T$ be a translation of $E$; then $T$ acts on points of $E$, on subsets of $E$ and on partial partitions of $E$. Let $\eta \in A E\left(\Pi^{*}(E)\right)$ be given by $\eta(\pi)=\pi \wedge T(\pi)$. Then $\rho(\eta)$ and $\theta(\eta)$ are given by setting for any $B \in \mathcal{P}(E)$ :

$$
\rho(\eta)(B)=\mathbf{1}_{B \cap T(B)} \quad \text { and } \quad \theta(\eta)(B)=\mathbf{1}_{B \cap T(B)} \cup \mathbf{1}_{B \backslash T(B)} .
$$

2. Let $\gamma$ be an opening on $\mathcal{P}(E)$. We define the operator $\bar{\gamma}$ on $\Pi^{*}(E)$ by $\bar{\gamma}(\pi)=\pi \wedge \mathbf{1}_{\gamma(\operatorname{supp}(\pi))}$. Then $\bar{\gamma}$ is an opening on $\Pi^{*}(E)$, characterized by its invariance domain

$$
\operatorname{lnv}(\bar{\gamma})=\left\{\pi \in \Pi^{*}(E) \mid \operatorname{supp}(\pi) \in \operatorname{lnv}(\gamma)\right\}
$$

Now $\rho(\bar{\gamma})$ is the map $B \mapsto \mathbf{1}_{\gamma(B)}$ and $\beta(\rho(\bar{\gamma}))$ operates on a partial partition by applying $\gamma$ to each block, and keeping the non-void opened blocks:

$$
\forall \pi \in \Pi^{*}(E), \quad \beta(\rho(\bar{\gamma}))(\pi)=\{\gamma(B) \mid B \in \pi, \gamma(B) \neq \emptyset\} .
$$

Then $\beta(\rho(\bar{\gamma}))$ is also an opening on $\Pi^{*}(E)$. We illustrate the behaviour of $\bar{\gamma}$ and $\beta(\rho(\bar{\gamma}))$ in Figure 9. Note that $\theta(\bar{\gamma})$ is the map $B \mapsto \mathbf{1}_{B \cap \gamma(E)}$.

This second example introduces a special case of set splitting operators: 

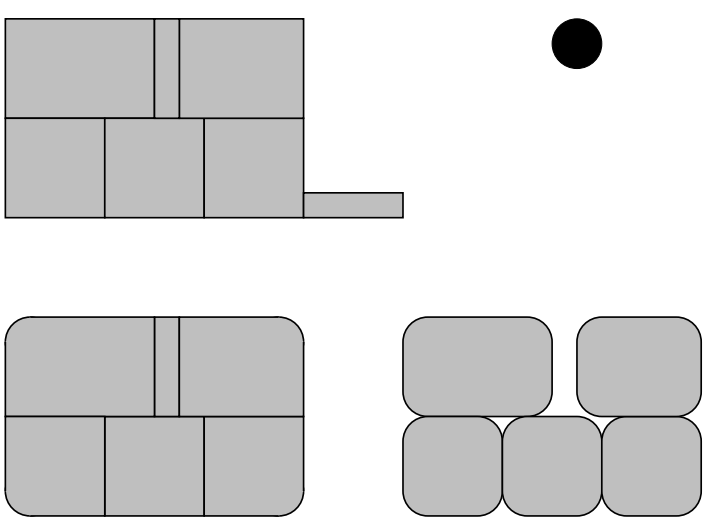

Fig. 9 Top left: a partial partition $\pi$. Top right: let $\gamma$ be the opening by the disk structuring element shown here. Bottom left: $\bar{\gamma}(\pi)$, the corners of $\operatorname{supp}(\pi)$ are rounded, and blocks included in narrow portions of $\operatorname{supp}(\pi)$ disappear. Bottom right: $\beta(\rho(\bar{\gamma}))(\pi)$, the corners of each block of $\pi$ are rounded, and all narrow blocks are removed.

Definition 11 1. Given an anti-extensive operator $\psi$ on $\mathcal{P}(E)$, the map $\mathbf{1}_{\psi}$ : $\mathcal{P}(E) \rightarrow \Pi^{*}(E): X \mapsto \mathbf{1}_{\psi(X)} \in \Pi^{*}(X)$ is called a set shrinking operator on $\mathcal{P}(E)$, and $\beta\left(\mathbf{1}_{\psi}\right)$ is called the block shrinking operator on $\Pi^{*}(E)$ derived from $\psi$.

2. [31] Given an operator $\psi$ on $\mathcal{P}(E)$, the blockwise extension of $\psi$ is the operator $\mathrm{B}(\psi)$ on $\Pi^{*}(E)$ given by $\mathrm{B}(\psi)(\pi)=\bigvee_{B \in \pi} \mathbf{1}_{\psi(B)}$ for all $\pi \in$ $\Pi^{*}(E)$.

We have then the following analogue of Proposition 5.

Proposition 12 For any anti-extensive operator $\psi$ on $\mathcal{P}(E)$ :

1. $\mathrm{B}(\psi)=\beta\left(\mathbf{1}_{\psi}\right)$ and for any $\pi \in \Pi^{*}(E)$,

$$
\mathrm{B}(\psi)(\pi)=\{\psi(B) \mid B \in \pi, \psi(B) \neq \emptyset\} ;
$$

2. for any $A \in \mathcal{P}(E), \mathrm{B}(\psi)\left(\mathbf{1}_{A}\right)=\mathbf{1}_{\psi(A)}$;

3. for any $p \in E$ and $\pi \in \Pi^{*}(E)$,

$$
\mathrm{Cl}_{\mathrm{B}(\psi)(\pi)}(p)= \begin{cases}\psi\left(\mathrm{Cl}_{\pi}(p)\right) & \text { if } p \in \psi\left(\mathrm{Cl}_{\pi}(p)\right) \\ \emptyset & \text { otherwise }\end{cases}
$$

4. $\mathrm{B}(\psi)$ is anti-extensive;

5. $\mathrm{B}(\psi)$ is isotone iff $\psi$ is isotone.

The map $A E(\mathcal{P}(E)) \rightarrow A E\left(\Pi^{*}(E)\right): \psi \mapsto \mathrm{B}(\psi)$ is an injective erosion and a monoid morphism.

Proof 1. By definition,

$$
\beta\left(\mathbf{1}_{\psi}\right)(\pi)=\bigcup_{B \in \pi} \mathbf{1}_{\psi}(B)=\bigcup_{B \in \pi} \mathbf{1}_{\psi(B)}=\bigvee_{B \in \pi} \mathbf{1}_{\psi(B)}=\mathrm{B}(\psi)(\pi),
$$

so $\mathrm{B}(\psi)=\beta\left(\mathbf{1}_{\psi}\right)$. Now $\mathbf{1}_{\psi(B)}=\{\psi(B)\}$ if $\psi(B) \neq \emptyset$, and is void otherwise. Thus $\bigcup_{B \in \pi} \mathbf{1}_{\psi(B)}$ gives $(17)$.

2. This follows from item 1 of Proposition 5 . 
3. By item 2 of Proposition $5, \mathrm{Cl}_{\mathrm{B}(\psi)(\pi)}(p)=\mathrm{Cl}_{\mathbf{1}_{\psi}\left(\mathrm{Cl}_{\pi}(p)\right)}(p)$, that is, the class of $p$ in $\mathbf{1}_{\psi\left(\mathrm{Cl}_{\pi}(p)\right)}$, which gives $\psi\left(\mathrm{Cl}_{\pi}(p)\right)$ if $p \in \psi\left(\mathrm{Cl}_{\pi}(p)\right)$ and $\emptyset$ otherwise.

4. This follows from item 3 of Proposition 5.

5. Given $X, Y \in \mathcal{P}(E)$ such that $X \subseteq Y$, we have $\psi(X) \subseteq \psi(Y) \Leftrightarrow$ $\mathbf{1}_{\psi(X)} \leq \mathbf{1}_{\psi(Y)}$ (since $\mathbf{1}$ • is an order-embedding). Thus $\psi$ is isotone iff $\mathbf{1}_{\psi}$ is isotone, and by item 4 of Proposition 5, this is equivalent to $\mathrm{B}(\psi)$ being isotone.

Since 1. : $A \mapsto \mathbf{1}_{A}$ is injective, the map $\psi \mapsto \mathbf{1}_{\psi}$ is injective. Now $\beta$ is injective by Proposition 5 , so $\psi \mapsto \beta\left(\mathbf{1}_{\psi}\right)=\mathrm{B}(\psi)$ is injective. Since $\mathbf{1}_{\text {• }}$ is an erosion, cf. (7), the map $A E(\mathcal{P}(E)) \rightarrow \Sigma(E): \psi \mapsto \mathbf{1}_{\psi}$ is an erosion (this works also for the empty infimum, or greatest element: id, the greatest element of $A E(\mathcal{P}(E))$, is mapped on $\mathbf{1}_{\bullet}$, the greatest element of $\left.\Sigma(E)\right)$. But $\beta: \Sigma(E) \rightarrow$ $A E\left(\Pi^{*}(E)\right)$ is also an erosion by Proposition 5. Thus $\psi \mapsto \beta\left(\mathbf{1}_{\psi}\right)=\mathrm{B}(\psi)$ is the composition of two erosions, hence an erosion.

For $\psi_{1}, \psi_{2} \in A E(\mathcal{P}(E))$, it remarked was in [31] that they satisfy the two properties $\psi_{i}(\emptyset)=\emptyset$ and $[X \cap Y=\emptyset] \Rightarrow\left[\psi_{i}(X) \cap \psi_{i}(Y)=\emptyset\right]$, and it was shown that these two properties guarantee that $\mathrm{B}\left(\psi_{1}\right) \mathrm{B}\left(\psi_{2}\right)=\mathrm{B}\left(\psi_{1} \psi_{2}\right)$. Given id the identity on $\mathcal{P}(E)$, clearly $\mathrm{B}(\mathbf{i d})$ is the identity on $\Pi^{*}(E)$. Therefore $\psi \mapsto \mathrm{B}(\psi)$ is a monoid morphism.

From now on we will write $\mathrm{B}(\psi)$ for $\beta\left(\mathbf{1}_{\psi}\right)$.

Given a sequence $x_{n}, n \in \mathbf{N}$, we write $x_{n} \downarrow x$ if the sequence $x_{n}$ is decreasing $\left(x_{n} \geq x_{n+1}\right)$ and $x=\bigwedge_{n \in \mathbf{N}} x_{n}$; an isotone operator $\psi$ is said to be $\downarrow$-continuous if $x_{n} \downarrow x \Rightarrow \psi\left(x_{n}\right) \downarrow \psi(x)$ [18].

Corollary 13 Let $\psi$ be $a \downarrow$-continuous isotone and anti-extensive operator on $\mathcal{P}(E)$. Then $\mathrm{B}(\psi)$ is a $\downarrow$-continuous isotone and anti-extensive operator on $\Pi^{*}(E)$. For $\gamma=\bigwedge_{n \geq 1} \psi^{n}, \gamma$ is the greatest opening $\leq \psi, \mathrm{B}(\gamma)=\bigwedge_{n \geq 1} \mathrm{~B}(\psi)^{n}$, and $\mathrm{B}(\gamma)$ is the greatest opening $\leq \mathrm{B}(\psi)$.

Proof $\mathrm{B}(\psi)$ is isotone by item 4 of Proposition 12. Take $\pi_{n} \downarrow \pi$ in $\Pi^{*}(E)$. By (2) and (3), for any $p \in E$ we have $\mathrm{Cl}_{\pi_{n}}(p) \downarrow \mathrm{Cl}_{\pi}(p)$; as $\psi$ is $\downarrow$-continuous, $\psi\left(\mathrm{Cl}_{\pi_{n}}(p)\right) \downarrow \psi\left(\mathrm{Cl}_{\pi}(p)\right)$. Now we have two cases:

1. For all $n \in \mathbf{N}, p \in \psi\left(\mathrm{Cl}_{\pi_{n}}(p)\right)$; then $p \in \bigwedge_{n \in \mathbf{N}} \psi\left(\mathrm{Cl}_{\pi_{n}}(p)\right)=\psi\left(\mathrm{Cl}_{\pi}(p)\right)$; by item 3 of Proposition $12, \mathrm{Cl}_{\mathrm{B}(\psi)\left(\pi_{n}\right)}(p)=\psi\left(\mathrm{Cl}_{\pi_{n}}(p)\right)$ for all $n \in \mathbf{N}$, and $\mathrm{Cl}_{\mathrm{B}(\psi)(\pi)}(p)=\psi\left(\mathrm{Cl}_{\pi}(p)\right)$. Hence $\mathrm{Cl}_{\mathrm{B}(\psi)\left(\pi_{n}\right)}(p) \downarrow \mathrm{Cl}_{\mathrm{B}(\psi)(\pi)}(p)$.

2. There is some $n \in \mathbf{N}$ such that $p \notin \psi\left(\mathrm{Cl}_{\pi_{n}}(p)\right)$; then $p \notin \psi\left(\mathrm{Cl}_{\pi_{m}}(p)\right)$ for all $m \geq n$, and $p \notin \psi\left(\mathrm{Cl}_{\pi}(p)\right)$; by item 3 of Proposition $12, \mathrm{Cl}_{\mathrm{B}(\psi)\left(\pi_{m}\right)}(p)=\emptyset$ for all $m \geq n$, and $\mathrm{Cl}_{\mathrm{B}(\psi)(\pi)}(p)=\emptyset$. As $\emptyset \downarrow \emptyset$, we get $\mathrm{Cl}_{\mathrm{B}(\psi)\left(\pi_{n}\right)}(p) \downarrow$ $\mathrm{Cl}_{\mathrm{B}(\psi)(\pi)}(p)$.

Since $\mathrm{Cl}_{\mathrm{B}(\psi)\left(\pi_{n}\right)}(p) \downarrow \mathrm{Cl}_{\mathrm{B}(\psi)(\pi)}(p)$ for any $p \in E$, applying again (2) and (3), we get $\mathrm{B}(\psi)\left(\pi_{n}\right) \downarrow \mathrm{B}(\psi)(\pi)$. Therefore $\mathrm{B}(\psi)$ is a $\downarrow$-continuous.

Let $\gamma=\bigwedge_{n>1} \psi^{n}$. By Proposition 12, the map $\psi \mapsto \mathrm{B}(\psi)$ is compatible with the infimum and with the composition, hence $\mathrm{B}(\gamma)=\bigwedge_{n>1} \mathrm{~B}(\psi)^{n}$. The fact that $\gamma$ and $\mathrm{B}(\gamma)$ are the greatest openings $\leq \psi$ and $\leq \mathrm{B}(\psi)$ respectively, follows from [18]. 
Remark 14 If we restrict ourselves to operators on $\Pi(E)$, hence to supportpreserving set / block splitting operators, we may not consider $\mathbf{1}_{A}$, but $\mathbf{1}_{A} \cup$ $\mathbf{0}_{E \backslash A}=\mathbf{1}_{A} \vee \mathbf{0}_{E}$. Thus item 1 of Proposition 5 becomes: $\beta(\sigma)\left(\mathbf{1}_{A} \vee \mathbf{0}_{E}\right)=$ $\sigma(A) \vee \mathbf{0}_{E}$. Then we have to replace $\rho$ by $\rho^{\prime}$ defined as follows:

$$
\rho^{\prime}(\eta): \mathcal{P}(E) \rightarrow \Pi(E): B \mapsto\left[\eta\left(\mathbf{1}_{B} \vee \mathbf{0}_{E}\right)\right]_{B}
$$

For an anti-extensive operator $\psi$ on $\mathcal{P}(E)$, instead of $\mathbf{1}_{\psi}$, we consider the map $\mathbf{1}_{\psi} \vee \mathbf{0}_{\bullet}: X \mapsto \mathbf{1}_{\psi(X)} \vee \mathbf{0}_{X}$. Then

$$
\beta\left(\mathbf{1}_{\psi} \vee \mathbf{0}_{\bullet}\right)(\pi)=\{\psi(B) \mid B \in \pi, \psi(B) \neq \emptyset\} \vee \mathbf{0}_{E}
$$

Up to these modifications, the above results remain valid for $\Pi(E)$ in place of $\Pi^{*}(E)$.

3.2 Partial connections and block splitting openings

It is known since [37] that given a connection $\mathcal{C}$, the operator on partitions that splits each block into its $\mathcal{C}$-components is an opening. This result led to further works on the relation between connections and partitions [39], then between partial connections and partial partitions [30]. Here we extend these studies by showing that a block splitting operator is an opening iff it is the one decomposing each block into its $\mathcal{C}$-components for some partial connection $\mathcal{C}$; these block splitting openings constitute a complete sublattice of the lattice of openings on $\Pi^{*}(E)$, isomorphic to the lattice of partial connections.

Given a partial connection $\mathcal{C}$, for any $A \in \mathcal{P}(E)$, recall the partial partition

$$
\mathrm{PC}^{\mathcal{C}}(A)=\left\{\gamma_{p}(A) \mid p \in A, \gamma_{p}(A) \neq \emptyset\right\}
$$

of all $\mathcal{C}$-components of $A$ (cf. Proposition 1$)$; note that $\operatorname{PC}^{\mathcal{C}}(A)$ is isotone in $A$, and commutes with the supremum on $\mathcal{C}$ (Proposition 2).

For any family $\mathcal{C} \subseteq \mathcal{P}(E)$, let

$$
\text { and } \quad \begin{aligned}
\Pi(E, \mathcal{C}) & =\Pi(E) \cap \mathcal{P}(\mathcal{C} \backslash\{\emptyset\}) \\
\Pi^{*}(E, \mathcal{C}) & =\Pi^{*}(E) \cap \mathcal{P}(\mathcal{C} \backslash\{\emptyset\}),
\end{aligned}
$$

be the families respectively of partitions and of partial partitions, whose blocks belong to $\mathcal{C}$ (in fact, blocks are non-void, so they belong to $\mathcal{C} \backslash\{\emptyset\}$ ). Then:

Proposition 15 Let $\mathcal{C} \subseteq \mathcal{P}(E)$ such that $\emptyset \in \mathcal{C}$. Then:

- $\mathcal{C}$ is a partial connection on $\mathcal{P}(E)$ iff $\Pi^{*}(E, \mathcal{C})$ is a dual Moore family of $\Pi^{*}(E)$ [30].

$-\mathcal{C}$ is a connection on $\mathcal{P}(E)$ iff $\Pi(E, \mathcal{C})$ is a dual Moore family of $\Pi(E)$ [39]. 
More characterizations can be found in [30]. Now the dual Moore family $\Pi^{*}(E, \mathcal{C})$ (or $\left.\Pi(E, \mathcal{C})\right)$ is the invariance domain of an opening. Given a partial connection $\mathcal{C}$, let $\mathrm{CS}^{\mathcal{C}}$ be the operator on $\Pi^{*}(E)$ that splits each block of a partial partition into its $\mathcal{C}$-components:

$$
\begin{aligned}
\forall \pi \in \Pi^{*}(E), \quad \mathrm{CS}^{\mathcal{C}}(\pi) & =\bigcup_{C \in \pi} \operatorname{PC}^{\mathcal{C}}(C) \\
& =\left\{\gamma_{p}(C) \mid C \in \pi, p \in C, \gamma_{p}(C) \neq \emptyset\right\} .
\end{aligned}
$$

The map $\mathrm{PC}^{\mathcal{C}}: \mathcal{P}(E) \rightarrow \Pi^{*}(E): A \mapsto \mathrm{PC}^{\mathcal{C}}(A)$ is a set splitting operator; when $\mathcal{C}$ is a connection, $\mathrm{PC}^{\mathcal{C}}$ is support-preserving, since $\mathrm{PC}^{\mathcal{C}}(A) \in \Pi(A)$. By $(20), \mathrm{CS}^{\mathcal{C}}=\beta\left(\mathrm{PC}^{\mathcal{C}}\right)$. Proposition 1 and item 2 of Proposition 5 give:

$$
\forall \pi \in \Pi^{*}(E), \forall p \in E, \quad \mathrm{Cl}_{\mathrm{CS}^{\mathcal{C}}(\pi)}(p)=\mathrm{Cl}_{\mathrm{PC}^{\mathcal{C}}\left(\mathrm{Cl}_{\pi}(p)\right)}(p)=\gamma_{p}\left(\mathrm{Cl}_{\pi}(p)\right) .
$$

Proposition 16 [30] For any partial connection $\mathcal{C}$ on $\mathcal{P}(E), \mathrm{CS}^{\mathcal{C}}$ is an opening on $\Pi^{*}(E)$, whose invariance domain is $\Pi^{*}(E, \mathcal{C})$. When $\mathcal{C}$ is a connection, the restriction of $\mathrm{CS}^{\mathcal{C}}$ to $\Pi(E)$ is an opening whose invariance domain is $\Pi(E, \mathcal{C})$.

In order to characterize $\mathrm{CS}^{\mathcal{C}}$ as a block splitting operator $\beta(\sigma)$ which is an opening, we analyse the domain of invariance of $\beta(\sigma)$ :

Definition 17 For any set splitting operator $\sigma$ on $\mathcal{P}(E)$, its fixed set is

$$
\mathcal{F}(\sigma)=\left\{X \in \mathcal{P}(E) \mid \sigma(X)=\mathbf{1}_{X}\right\} .
$$

Proposition 18 For any set splitting operator $\sigma$ on $\mathcal{P}(E)$, we have $\emptyset \in \mathcal{F}(\sigma)$ and $\operatorname{lnv}(\beta(\sigma))=\Pi^{*}(E, \mathcal{F}(\sigma))$. If $\sigma$ is support-preserving, then $\mathcal{S}(E) \subseteq \mathcal{F}(\sigma)$ and the restriction of $\beta(\sigma)$ to $\Pi(E)$ has invariance domain $\Pi(E, \mathcal{F}(\sigma))$.

Proof As $\sigma(\emptyset)=\varnothing=\mathbf{1}_{\emptyset}, \emptyset \in \mathcal{F}(\sigma)$. Let $\pi \in \Pi^{*}(E)$. Since $\beta(\sigma(\pi)) \leq \pi$, by (11) we have $\beta(\sigma(\pi))=\pi$ iff for every $B \in \pi,[\beta(\sigma)(\pi)]_{B}=[\pi]_{B}$, in other words by (14), $\sigma(B)=[\pi]_{B}=\mathbf{1}_{B}$, that is $B \in \mathcal{F}(\sigma)$. Thus $\operatorname{lnv}(\beta(\sigma))=$ $\Pi^{*}(E, \mathcal{F}(\sigma))$.

Let $\sigma$ be support-preserving. For any $p \in E, \sigma(\{p\}) \in \Pi^{*}(\{p\})=\left\{\varnothing, \mathbf{1}_{\{p\}}\right\}$ with $\operatorname{supp}(\sigma(\{p\}))=\{p\}$, thus $\sigma(\{p\})=\mathbf{1}_{\{p\}}$, hence $\{p\} \in \mathcal{F}(\sigma)$. Restricting $\beta(\sigma)$ to $\Pi(E)$, its invariance domain is $\Pi^{*}(E, \mathcal{F}(\sigma)) \cap \Pi(E)=\Pi(E, \mathcal{F}(\sigma))$.

Lemma 19 For any partial connection $\mathcal{C}, \mathcal{F}\left(\mathrm{PC}^{\mathcal{C}}\right)=\mathcal{C}$

Proof Indeed, $A \in \mathcal{F}\left(\mathrm{PC}^{\mathcal{C}}\right)$ iff $\mathrm{PC}^{\mathcal{C}}(A)=\mathbf{1}_{A}$; clearly $\mathrm{PC}^{\mathcal{C}}(\emptyset)=\emptyset=\mathbf{1}_{\emptyset}$ and $\emptyset \in \mathcal{C}$; now for $A \neq \emptyset, \mathrm{PC}^{\mathcal{C}}(A)=\mathbf{1}_{A}$ iff $A$ is a $\mathcal{C}$-component of $A$, that is, $A \in \mathcal{C}$.

Theorem 20 Let $\sigma$ be an isotone set splitting operator on $\mathcal{P}(E)$. Then: 
1. $\mathcal{F}(\sigma)$ is a partial connection and $\mathrm{CS}^{\mathcal{F}(\sigma)}$ is the greatest opening $\leq \beta(\sigma)$ (on $\left.\Pi^{*}(E)\right)$. In particular, $\beta(\sigma)$ is an opening iff there is a partial connection $\mathcal{C}$ such that $\beta(\sigma)=\mathrm{CS}^{\mathcal{C}}$, in other words $\sigma=\mathrm{PC}^{\mathcal{C}}$; we have then necessarily $\mathcal{C}=\mathcal{F}(\sigma)$.

2. If $\sigma$ is support-preserving, then $\mathcal{F}(\sigma)$ is a connection and:

- in the lattice of operators on $\Pi(E), \mathrm{CS}^{\mathcal{F}(\sigma)}$ is the greatest opening $\leq$ $\beta(\sigma)$;

- in the lattice of operators on $\Pi^{*}(E), \mathrm{CS}^{\mathcal{F}(\sigma)}$ is the greatest block splitting operator on $\Pi^{*}(E)$ inducing on $\Pi(E)$ an opening $\leq \beta(\sigma)$.

In particular, $\beta(\sigma)$ induces an opening on $\Pi(E)$ iff there is a connection $\mathcal{C}$ such that $\beta(\sigma)=\mathrm{CS}^{\mathcal{C}}$, in other words $\sigma=\mathrm{PC}^{\mathcal{C}}$; we have then necessarily $\mathcal{C}=\mathcal{F}(\sigma)$.

Proof 1. Since $\sigma$ is isotone, $\beta(\sigma)$ is isotone and anti-extensive (Proposition 5). It is then known [16] that $\operatorname{Inv}(\beta(\sigma))$ is a dual Moore family, and that the greatest opening $\leq \beta(\sigma)$ is the one having the same invariance domain $\operatorname{lnv}(\beta(\sigma))$. By Proposition $18, \operatorname{Inv}(\beta(\sigma))=\Pi^{*}(E, \mathcal{F}(\sigma))$. As $\Pi^{*}(E, \mathcal{F}(\sigma))$ is a dual Moore family, $\mathcal{F}(\sigma)$ is a partial connection by Proposition 15 . The greatest opening $\leq \beta(\sigma)$ is the one whose invariance domain is $\operatorname{Inv}(\beta(\sigma))=\Pi^{*}(E, \mathcal{F}(\sigma))$, by Proposition 16 this opening is $\operatorname{CS}^{\mathcal{F}(\sigma)}$. If $\beta(\sigma)$ is an opening, then it is the greatest one $\leq \beta(\sigma)$, thus $\beta(\sigma)=\mathrm{CS}^{\mathcal{F}(\sigma)}=\beta\left(\mathrm{PC}^{\mathcal{F}(\sigma)}\right)$, and as $\beta$ injective (Proposition 5), we get $\sigma=\mathrm{PC}^{\mathcal{F}(\sigma)}$. Conversely, if for a partial connection $\mathcal{C}$ we have $\beta(\sigma)=\mathrm{CS}^{\mathcal{C}}$, that is, $\sigma=\mathrm{PC}^{\mathcal{C}}$, then $\beta(\sigma)$ is an opening by Proposition 16 , and $\mathcal{C}=\mathcal{F}(\sigma)$ by Lemma 19 .

2. Since $\sigma$ is support-preserving, by Proposition $18 \mathcal{F}(\sigma)$ comprises all singletons; as it is partial connection, it will be a connection.

Let us first restrict ourselves to operators on $\Pi(E)$. Here $\operatorname{lnv}(\beta(\sigma))=$ $\Pi(E, \mathcal{F}(\sigma))$ is a dual Moore family, and the greatest opening $\leq \beta(\sigma)$ is the one having the same invariance domain $\Pi(E, \mathcal{F}(\sigma))$; by Proposition 16 , this opening is $\operatorname{CS}^{\mathcal{F}(\sigma)}$.

Let us now consider again operators on $\Pi^{*}(E)$. Clearly $\operatorname{CS}^{\mathcal{F}(\sigma)}$ is a block splitting operator on $\Pi^{*}(E)$, and by the previous paragraph it induces on $\Pi(E)$ an opening $\leq \beta(\sigma)$. Let $\zeta$ be a set splitting operator such that $\beta(\zeta)$ induces on $\Pi(E)$ an opening $\leq \beta(\sigma)$. By the previous paragraph, the restriction of $\beta(\zeta)$ to $\Pi(E)$ is $\leq \operatorname{CS}^{\mathcal{F}(\sigma)}$. Thus for $\pi \in \Pi(E), \beta(\zeta)(\pi) \leq \operatorname{CS}^{\mathcal{F}(\sigma)}(\pi)$. Now for every $B \in \mathcal{P}(E) \backslash\{\emptyset\}$ there is some $\pi \in \Pi(E)$ with $B \in \pi$, hence by $(11,14)$ we get:

$$
\zeta(B)=[\beta(\zeta)(\pi)]_{B} \leq\left[\mathrm{CS}^{\mathcal{F}(\sigma)}(\pi)\right]_{B}=\left[\beta\left(\mathrm{PC}^{\mathcal{F}(\sigma)}\right)(\pi)\right]_{B}=\mathrm{PC}^{\mathcal{F}(\sigma)}(B) .
$$

Thus by (13), for any $\pi \in \Pi^{*}(E)$ we have:

$$
\beta(\zeta)(\pi)=\bigvee_{B \in \pi} \zeta(B) \leq \bigvee_{B \in \pi} \operatorname{PC}^{\mathcal{F}(\sigma)}(B)=\operatorname{CS}^{\mathcal{F}(\sigma)}(\pi)
$$

Therefore $\beta(\zeta) \leq \mathrm{CS}^{\mathcal{F}(\sigma)}$ on $\Pi^{*}(E)$, and $\operatorname{CS}^{\mathcal{F}(\sigma)}$ is the greatest block splitting operator on $\Pi^{*}(E)$ inducing on $\Pi(E)$ an opening $\leq \beta(\sigma)$. If $\beta(\sigma)$ induces 
an opening on $\Pi(E)$, then $\beta(\sigma) \leq \operatorname{CS}^{\mathcal{F}(\sigma)}$, but $\operatorname{CS}^{\mathcal{F}(\sigma)} \leq \beta(\sigma)$ by item 1 , thus $\beta(\sigma)=\mathrm{CS}^{\mathcal{F}(\sigma)}$, in other words $\sigma=\mathrm{PC}^{\mathcal{F}(\sigma)}$ (by the injectivity of $\beta$ ). Conversely, if for a connection $\mathcal{C}$ we have $\beta(\sigma)=\mathrm{CS}^{\mathcal{C}}$, that is, $\sigma=\mathrm{PC}^{\mathcal{C}}$, then $\beta(\sigma)$ is an opening by Proposition 16 , and $\mathcal{C}=\mathcal{F}(\sigma)$ by Lemma 19 .

Thus, given an isotone set splitting operator $\sigma$ such that $\beta(\sigma)$ is an opening, for any $A \in \mathcal{P}(E) \backslash\{\emptyset\}$, the $\mathcal{F}(\sigma)$-components of $A$ are the blocks of $\sigma(A)=$ $\mathrm{PC}^{\mathcal{F}(\sigma)}(A)$.

A typical example of block splitting opening $\mathrm{CS}^{\mathcal{C}}$ arises when we choose for $\mathcal{C}$ a usual connection in the Euclidean or digital space: we split each block into its connected components. We can also use the derived connections of Figures 3 and 4 (or even the one of Figure 5). However, there are many more types of partial connections, many examples were given in $[36,29,17,5,30]$. Let us indeed describe some atypic instances of block splitting openings:

- Let $\gamma$ be an opening on $\mathcal{P}(E)$. Then $\mathrm{B}(\gamma)$ is a block shrinking opening, and (17) gives for any $\pi \in \Pi^{*}(E)$ :

$$
\mathrm{B}(\gamma)(\pi)=\{\gamma(B) \mid B \in \pi, \gamma(B) \neq \emptyset\}
$$

cf. Figure 9. Here $\mathcal{F}\left(\mathbf{1}_{\gamma}\right)=\operatorname{Inv}(\gamma)$, it is a dual Moore family of $\mathcal{P}(E)$, hence a partial connection such that every subset $B$ of $E$ has at most one $\operatorname{lnv}(\gamma)$-component: $\gamma(B)$ if $\gamma(B) \neq \emptyset$, and none if $\gamma(B)=\emptyset[30]$.

- More generally, let $\psi$ be an isotone anti-extensive operator on $\mathcal{P}(E)$. We have $\mathcal{F}\left(\mathbf{1}_{\psi}\right)=\operatorname{Inv}(\psi)$, it is a dual Moore family of $\mathcal{P}(E)$, hence a partial connection. Let $\gamma$ be the opening on $\mathcal{P}(E)$ given by $\operatorname{lnv}(\gamma)=\operatorname{lnv}(\psi)$; then $\gamma$ is the greatest opening on $\mathcal{P}(E)$ which is $\leq \psi$, and $\mathrm{B}(\gamma)=\mathrm{CS}^{\mathcal{F}\left(\mathbf{1}_{\psi}\right)}$ is the greatest opening on $\Pi^{*}(E)$ which is $\leq \mathrm{B}(\psi)$. If $\psi$ is $\downarrow$-continuous, we can obtain $\mathrm{B}(\gamma)$ as the limit of the iteration of $\mathrm{B}(\psi)$, cf. Corollary 13 .

- Let $\mathcal{C}=\{\emptyset\} \cup \mathcal{S}(E)$, the least connection. The $\mathcal{C}$-components of a set are its singletons, so $\mathrm{CS}^{\mathcal{C}}$ is the block grinding opening $[30,31]$ that pulverizes each block into its singletons: $\operatorname{CS}^{\mathcal{C}}(\pi)=\mathbf{0}_{\text {supp }(\pi)}$.

- For a fixed non-void $\pi_{0} \in \Pi^{*}(E)$, take the block splitting opening of Example 6, namely $\sigma: B \mapsto \mathbf{1}_{B} \wedge \pi_{0}$ and $\beta(\sigma): \pi \mapsto \pi \wedge \pi_{0}$. Here $\mathcal{F}(\sigma)$ is the partial connection made of all subsets of blocks of $\pi_{0}$, we write it $\operatorname{subbl}\left(\pi_{0}\right)[30]$; thus:

$$
\operatorname{subbl}\left(\pi_{0}\right)=\bigcup_{B \in \pi_{0}} \mathcal{P}(B)=\left\{X \in \mathcal{P}(E) \mid \exists B \in \pi_{0}, X \subseteq B\right\}
$$

For any $A \in \mathcal{P}(E) \backslash\{\emptyset\}$, the $\mathcal{F}(\sigma)$-components of $A$ are the non-void $A \cap B$ for $B \in \pi_{0}$. When $\pi_{0} \in \Pi(E), \operatorname{subbl}\left(\pi_{0}\right)$ is a connection; it was already described in [36].

Given a set splitting operator $\sigma$, we see from Propositions 5 and 18 that $\beta(\sigma)$ is an opening iff $\sigma$ is isotone and for any $A \in \mathcal{P}(E) \backslash\{\emptyset\}$ we have $\sigma(A) \in \Pi^{*}(E, \mathcal{F}(\sigma))$, in other words all blocks of $\sigma(A)$ belong to $\mathcal{F}(\sigma)$. By the above theorem, this is the necessary and sufficient condition for $\sigma$ to be, 
for some partial connection $\mathcal{C}$, the operator $\mathrm{PC}^{\mathcal{C}}$ splitting every set into the partial partition of its $\mathcal{C}$-components.

The above theorem has an important consequence, which in some way implicitly contains many of our results on partial connections:

Corollary 21 The set of block splitting openings is a complete sublattice of the complete lattice of openings on $\Pi^{*}(E)$, and by the correspondence $\mathcal{C} \leftrightarrow \mathrm{CS}^{\mathcal{C}}$ it is isomorphic to the complete lattice of partial connections on $\mathcal{P}(E)$.

Proof The least opening on $\Pi^{*}(E)$ is the constant $\pi \mapsto \varnothing$, it is the block splitting operator corresponding to the constant set splitting operator $X \mapsto \emptyset$. The greatest opening on $\Pi^{*}(E)$ is the identity, which is $\beta\left(\mathbf{1}_{\bullet}\right)$, with $\mathbf{1}_{\bullet}: X \mapsto$ $\mathbf{1}_{X}$. Consider a non-void family of set splitting operators $\sigma_{i}(i \in I \neq \emptyset)$, such that the $\beta\left(\sigma_{i}\right)$ are openings. Then $\bigvee_{i \in I} \beta\left(\sigma_{i}\right)$ is an openings $[35,16]$; but $\bigvee_{i \in I} \beta\left(\sigma_{i}\right)=\beta\left(\bigvee_{i \in I} \sigma_{i}\right)$ (by Proposition 5), so this opening is block splitting. The infimum, in the lattice of openings, of the $\beta\left(\sigma_{i}\right)$, is the greatest opening $\leq \bigwedge_{i \in I} \beta\left(\sigma_{i}\right)[35,16]$; but $\bigwedge_{i \in I} \beta\left(\sigma_{i}\right)=\beta\left(\bigwedge_{i \in I} \sigma_{i}\right)$, it is thus an anti-extensive block splitting operator; by Theorem 20 , the greatest opening $\leq \beta\left(\bigwedge_{i \in I} \sigma_{i}\right)$ is block splitting. Therefore the set of block splitting openings is a complete sublattice of the complete lattice of openings on $\Pi^{*}(E)$.

Consider two partial connections $\mathcal{C}$ and $\mathcal{C}^{\prime}$. If $\mathcal{C} \subseteq \mathcal{C}^{\prime}$, then $\operatorname{PC}^{\mathcal{C}}(X) \leq$ $\mathrm{PC}^{\mathcal{C}^{\prime}}(X)$ by Proposition 2 ; by $(20)$, we get $\operatorname{CS}^{\mathcal{C}}(\pi) \leq \operatorname{CS}^{\mathcal{C}^{\prime}}(\pi)$ for all $\pi \in \Pi^{*}(E)$, hence $\mathrm{CS}^{\mathcal{C}} \leq \mathrm{CS}^{\mathcal{C}^{\prime}}$. Conversely, if $\mathrm{CS}^{\mathcal{C}} \leq \mathrm{CS}^{\mathcal{C}^{\prime}}$, then for $C \in \mathcal{C} \backslash\{\emptyset\}$ we have $\mathbf{1}_{C}=\operatorname{CS}^{\mathcal{C}}\left(\mathbf{1}_{C}\right) \leq \operatorname{CS}^{\mathcal{C}^{\prime}}\left(\mathbf{1}_{C}\right) \leq \mathbf{1}_{C}$, which gives $\operatorname{CS}^{\mathcal{C}^{\prime}}\left(\mathbf{1}_{C}\right)=\mathbf{1}_{C}$, thus $C \in \mathcal{C}^{\prime}$; hence $\mathcal{C} \subseteq \mathcal{C}^{\prime}$. Therefore $\mathcal{C} \subseteq \mathcal{C}^{\prime} \Leftrightarrow \mathrm{CS}^{\mathcal{C}} \leq \mathrm{CS}^{\mathcal{C}^{\prime}}$, and the map $\mathcal{C} \mapsto \mathrm{CS}^{\mathcal{C}}$ is an isomorphism between the two complete lattices.

This gives a new light on Proposition 2, which states that for a fixed $A \in \mathcal{P}(E)$, the map $\mathcal{C} \mapsto \mathrm{PC}^{\mathcal{C}}(A)$ is a dilation; indeed, it expresses at the level of a single block $A$ the fact that for a supremum $\mathcal{C}=\operatorname{Con}^{*}\left(\bigcup_{i \in I} \mathcal{C}_{i}\right)$ of partial connections $\mathcal{C}_{i}$, the opening $\mathrm{CS}^{\mathcal{C}}$ is the supremum, in the lattice of operators, of the openings $\operatorname{CS}^{\mathcal{C}_{i}}$, in other words for any $\pi \in \Pi^{*}(E)$ we have $\operatorname{CS}^{\mathcal{C}}(\pi)=$ $\bigvee_{i \in I} \mathrm{CS}^{\mathcal{C}_{i}}(\pi)$. Just before Proposition 2, we said that for $I \neq \emptyset$, given the systems of partial connection openings $\left(\gamma_{p}^{i}, p \in E\right)$ of $\mathcal{C}_{i}$, the infimum $\bigcap_{i \in I} \mathcal{C}_{i}$ has its system of partial connection openings $\left(\gamma_{p}, p \in E\right)$ such that for every $p \in E, \gamma_{p}$ is the infimum, in the lattice of openings on $\mathcal{P}(E)$, of the $\gamma_{p}^{i}, i \in I$. Now for $\pi \in \Pi^{*}(E)$ and $p \in E$, by (21) we have $\mathrm{Cl}_{\mathrm{CS}^{c_{i}(\pi)}}(p)=\gamma_{p}^{i}\left(\mathrm{Cl}_{\pi}(p)\right)$, and the fact that $\mathrm{CS}^{\mathcal{C}_{i}}$ is an opening implies that $\gamma_{p}^{i}$ is an opening, thus the greatest opening below each $\mathrm{CS}^{\mathcal{C}_{i}}$ must correspond to the greatest opening below each $\gamma_{p}^{i}$.

Let us illustrate this result with an example. Take $E=\mathbf{Z}^{2}$, and for $\mathcal{C}_{\text {std }}$ the family of all 4-connected sets. Given a pair $H$ (resp., $V$ ) of horizontally (resp., vertically) adjacent pixels, following the method of Figure 3, we obtain the partial connections $\mathcal{H}=\mathcal{C}_{H}^{*}$ and $\mathcal{V}=\mathcal{C}_{V}^{*}$ consisting of all 4-connected subsets $X$ of $\mathbf{Z}^{2}$ such that $X \circ H=X$ (resp., $X \circ V=X$ ). Equivalently, $\mathcal{H}$ (resp., $\mathcal{V})$ consists of all 4-connected sets $X$ such that each $p \in X$ is horizontally 
(resp., vertically) adjacent to some $q \in X$. Now $\mathcal{C}=\operatorname{Con}^{*}(\mathcal{H} \cup \mathcal{V})$ is the family of all non-singleton 4-connected sets. We show in Figure 10 a partial partition $\pi$, then $\operatorname{CS}^{\mathcal{H}}(\pi), \operatorname{CS}^{\mathcal{V}}(\pi)$, and $\operatorname{CS}^{\mathcal{H}}(\pi) \vee \operatorname{CS}^{\mathcal{V}}(\pi)=\operatorname{CS}^{\mathcal{C}}(\pi)$; then $\pi^{\prime}=\operatorname{CS}^{\mathcal{H}}(\pi) \wedge \mathrm{CS}^{\mathcal{V}}(\pi)$ and $\pi^{\prime \prime}=\operatorname{CS}^{\mathcal{H}}\left(\pi^{\prime}\right) \wedge \mathrm{CS}^{\mathcal{V}}\left(\pi^{\prime}\right)$; since $\pi^{\prime \prime}$ is invariant under $\mathrm{CS}^{\mathcal{H}}$ and $\mathrm{CS}^{\mathcal{V}}$, we have $\pi^{\prime \prime}=\operatorname{CS}^{\mathcal{H} \cap \mathcal{V}}(\pi)$.
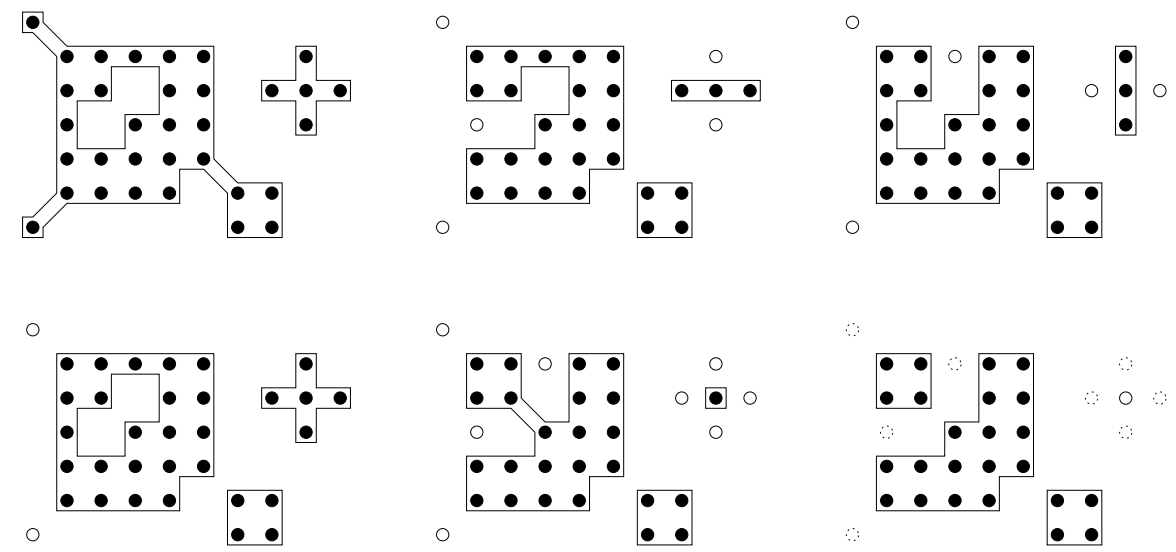

Fig. 10 Pixels in the support of a partial partition are shown as filled disks; those that are removed by an operator are shown as hollow circles (and those previously removed as dashed hollow circles). Each block is delineated by a polygon. Top left: $\pi$. Top center and right: $\operatorname{CS}^{\mathcal{H}}(\pi)$ and $\operatorname{CS}^{\mathcal{V}}(\pi)$, where $\mathcal{H}$ (resp., $\mathcal{V}$ ) is the partial connection of all 4-connected sets where each pixel has a horizontal (resp., vertical) neighbour. Bottom left: $\operatorname{CS}^{\mathcal{H}}(\pi) \vee \operatorname{CS}^{\mathcal{V}}(\pi)=$ $\operatorname{CS}^{\mathcal{C}}(\pi)$, where $\mathcal{C}=\operatorname{Con}^{*}(\mathcal{H} \cup \mathcal{V})$ is the partial connection of all non-singleton 4-connected sets. Bottom center: $\left(\mathrm{CS}^{\mathcal{H}} \wedge \mathrm{CS}^{\mathcal{V}}\right)(\pi)$ is not invariant under $\mathrm{CS}^{\mathcal{H}}$ and $\mathrm{CS}^{\mathcal{V}}$. Bottom right: $\left(\mathrm{CS}^{\mathcal{H}} \wedge \mathrm{CS}^{\mathcal{V}}\right)^{2}(\pi)$ is invariant under $\operatorname{CS}^{\mathcal{H}}$ and $\mathrm{CS}^{\mathcal{V}}$, so it is $\operatorname{CS}^{\mathcal{H} \cap \mathcal{V}}(\pi)$.

We saw above the example of the block splitting opening $\mathrm{B}(\gamma)$ for an opening $\gamma$ on $\mathcal{P}(E)$. In [31] we showed that for an adjunction $(\varepsilon, \delta)$ on $\mathcal{P}(E)$ where $\varepsilon(\emptyset)=\emptyset$ (or equivalently, $\forall X \in \mathcal{P}\left(E_{1}\right), X \neq \emptyset \Rightarrow \delta(X) \neq \emptyset$ ), defining $\mathrm{B}(\varepsilon)$ and $\mathrm{B}(\delta)$ according to Definition 11 , then $(\mathrm{B}(\varepsilon), \mathrm{B}(\delta))$ is an adjunction on $\Pi^{*}(E)$.

Proposition 22 Let $(\varepsilon, \delta)$ be an adjunction on $\mathcal{P}(E)$ such that $\varepsilon(\emptyset)=\emptyset$ (equivalently, $\left.\forall X \in \mathcal{P}\left(E_{1}\right), X \neq \emptyset \Rightarrow \delta(X) \neq \emptyset\right)$. Then $\mathrm{B}(\delta \varepsilon)=\mathrm{B}(\delta) \mathrm{B}(\varepsilon)$.

Proof By [31], B $(\varepsilon)$ satisfies (17), that is, for any $\pi \in \Pi^{*}(E)$ we have

$$
\mathrm{B}(\varepsilon)(\pi)=\{\varepsilon(B) \mid B \in \pi, \varepsilon(B) \neq \emptyset\} .
$$

Then by Definition 11 we get

$$
\mathrm{B}(\delta) \mathrm{B}(\varepsilon)(\pi)=\bigvee\left\{\mathbf{1}_{\delta \varepsilon(B)} \mid B \in \pi, \varepsilon(B) \neq \emptyset\right\} .
$$

By hypothesis, $\varepsilon(B) \neq \emptyset \Rightarrow \delta \varepsilon(B) \neq \emptyset$, and as $\delta$ is a dilation, $\delta(\emptyset)=\emptyset$; so $\varepsilon(B) \neq \emptyset \Leftrightarrow \delta \varepsilon(B) \neq \emptyset$, and in this case $\mathbf{1}_{\delta \varepsilon(B)}=\{\delta \varepsilon(B)\}$. Since $\delta \varepsilon$ is 
anti-extensive, the $\delta \varepsilon(B), B \in \pi$, are pairwise disjoint; also by Proposition 12, $\delta \varepsilon$ must satisfy (17). Therefore

$$
\begin{gathered}
\mathrm{B}(\delta) \mathrm{B}(\varepsilon)(\pi)=\bigvee\{\{\delta \varepsilon(B)\} \mid B \in \pi, \delta \varepsilon(B) \neq \emptyset\} \\
=\{\delta \varepsilon(B) \mid B \in \pi, \delta \varepsilon(B) \neq \emptyset\}=\mathrm{B}(\delta \varepsilon)(\pi),
\end{gathered}
$$

thus the two openings $\mathrm{B}(\delta \varepsilon)$ and $\mathrm{B}(\delta)(\mathrm{B}(\varepsilon)$ are equal.

\section{Conclusion}

This paper initiates the study of block splitting operators on the complete lattice of partial partitions. Section 3 explores a simple but fruitful idea: to make an anti-extensive operator on (partial) partitions by splitting each block independently; in particular we can apply to each block an anti-extensive operator on sets. This principle is explored quite generally in Subsection 3.1, where the lattice-theoretical and monoid features of set splitting and block splitting are investigated. The special case of isotone operators is also analysed.

The remainder of Section 3 rests on another simple but powerful idea: the archetype of set splitting is the decomposition of a set into its connected components according to a (partial) connection. Using the background of Subsection 2.2, Subsection 3.2 expands the findings of $[30,39]$ concerning the relations between partial connections and partial partitions; the main conclusion (cf. Theorem 20 and Corollary 21) is that a block splitting opening coincides with the splitting of blocks into $\mathcal{C}$-components for some partial connection $\mathcal{C}$, and this correspondence is a lattice isomorphism between partial connections and block splitting openings, the latter forming a complete sublattice of the lattice of all openings.

Subsection 3.2, together with [30], provides a theoretical basis for Serra's connective segmentation approach $[30,32,39]$. Here a criterion $\mathrm{cr}$ associates to each image $F$ (seen as function $E \rightarrow T$ ) and each subset $A$ of $E$ a binary value $\operatorname{cr}[F, A]$ that tells whether the set $A$ is homogeneous w.r.t. the function $F$. The basic assumption is that "a union of overlapping homogeneous sets must be homogeneous", in other words the set $\mathcal{C}_{\mathrm{cr}}^{F}=\{A \in \mathcal{P}(E) \mid \operatorname{cr}[F, A]=1\}$ must be a partial connection; we say then that the criterion or is partially connective. From this property the segmentation of the function $F$ on a subset $A$ of $E$ is given by the partial partition $\operatorname{PC}^{\mathcal{C}_{\mathrm{cr}}^{F}}(A)$ of all $\mathcal{C}_{\mathrm{cr}}^{F}$-components of $A$. The results obtained in Subsection 3.2 are relevant to this segmentation model, in particular on the question "when does a segmentation algorithm follow the connective model ?" Indeed, given a segmentation algorithm associating to each function $F$ and each subset $A$ of $E$ a partial partition $\sigma^{F}(A)$ of $A$, one has only to check that for every function $F$, the block splitting operator $\beta\left(\sigma^{F}\right)$ is an opening; equivalently, $\sigma^{F}$ must be isotone, and for every $A \in \mathcal{P}(E)$, all blocks of $\sigma^{F}(A)$ must belong to $\mathcal{F}\left(\sigma^{F}\right)$.

Often a segmentation algorithm only associates to a function $F$ a (partial) partition $\pi^{F}$ of the whole space $E$. Then, according to Example 6, we take 
the set splitting operator $\sigma: B \mapsto \mathbf{1}_{B} \wedge \pi^{F}$, giving rise to the block splitting opening $\beta(\sigma): \pi \mapsto \pi \wedge \pi^{F}$, corresponding to the partial connection $\operatorname{subbl}\left(\pi^{F}\right)$ consisting of all subsets of all blocks of $\pi^{F}$. Generally the space $E$ is provided with a standard connection $\mathcal{C}_{s t d}$, and we have $\pi^{F} \in \Pi^{*}\left(E, \mathcal{C}_{s t d}\right)$ (all blocks of $\pi^{F}$ are connected according to $\left.\mathcal{C}_{s t d}\right)$; then we take the partial connection $\operatorname{subbl}\left(\pi^{F}\right) \cap \mathcal{C}_{s t d}$, giving thus the set splitting operator $B \mapsto \mathrm{PC}^{\mathcal{C}_{\text {std }}}\left(\mathbf{1}_{B} \wedge \pi^{F}\right)$ and the block splitting opening $\pi \mapsto \operatorname{CS}^{\mathcal{C}_{\text {std }}}\left(\pi \wedge \pi^{F}\right)$ : we split every block according to $\pi^{F}$, then split the resulting blocks into their $\mathcal{C}_{s t d}$-components. Such a construction has been implicitly used in [39], cf. the discussion at the end of [30] on "a posteriori" connective criteria.

The second paper will study non-isotone idempotent block splitting operators, in particular those involved in some variants $[38,41]$ of connective segmentation.

Acknowledgements This work arose from several years if discussion with Jean Serra, and more recent ones with Pierre Soille.

\section{References}

1. Bauer, A., Birkedal, L., Scott, D.: Equilogical spaces. Theoretical Computer Science 315, 35-59 (2004)

2. Birkhoff, G.: Lattice Theory, American Mathematical Society Colloquium Publications, vol. 25, 8th printing, 3rd edn. American Mathematical Society, Providence, RI (1995)

3. Bloch, I., Heijmans, H., Ronse, C.: Mathematical Morphology. In: M. Aiello, I. PrattHartmann, J. van Benthem (eds.) Handbook of Spatial Logics, chap. 14, pp. 857-944. Springer (2007)

4. Blyth, T.: Lattices and Ordered Algebraic Structures. Springer, London (2005)

5. Braga-Neto, U., Goutsias, J.: A theoretical tour of connectivity in image processing and analysis. Journal of Mathematical Imaging and Vision 19(1), 5-31 (2003)

6. Davey, B., Priestley, H.: Introduction to Lattices and Order, 2nd edn. Cambridge University Press, Cambridge, UK (2002)

7. Denecke, K., Erné, M., Wismath, S. (eds.): Galois Connections and Applications. Kluwer, Dordrecht, NL (2004)

8. Draškovičová, H.: The lattice of partitions in a set. Acta Fac. Rerum Natur. Univ. Comenian. Math. Publ. 24, 37-65 (1970)

9. Draškovičová, H.: Congruence relations on the lattice of partitions in a set. Matematický Casopis (Slovenska Akademia Vied) 21, 141-153 (1971)

10. Dubreil, P., Dubreil-Jacotin, M.: Théorie algébriques des relations d'équivalence. Journal de Mathématiques Pures et Appliquées (Ser. IX) 18, 63-95 (1939)

11. Erné, M.: Closure. In: F. Mynard, E. Pearl (eds.) Beyond Topology, Contemporary Mathematics, vol. 486, pp. 163-238. American Mathematical Society (2009)

12. Erné, M., Vainio, R.: Connectivity in lattice-ordered spaces. Mathematische Nachrichten 147, 13-28 (1990)

13. Gierz, G., Hofmann, K., Keimel, K., Lawson, J., Mislove, M., Scott, D.: Continuous Lattices and Domains, Encyclopedia of Mathematics and its Applications, vol. 93. Cambridge University Press, Cambridge, UK (2003)

14. Grätzer, G.: General Lattice Theory, 2nd edn. Birkhäuser, Basel (2003)

15. Hadwiger, H.: Minkowskische Addition und Subtraktion beliebiger Punktmengen und die Theoreme von Erhard Schmidt. Math. Zeitschrift 53, 210-218 (1950)

16. Heijmans, H.: Morphological Image Operators. Advances in Electronics and Electron Physics Series. Academic Press, Boston (1994)

17. Heijmans, H.: Connected morphological operators for binary images. Computer Vision and Image Understanding 73(1), 99-120 (1999) 
18. Heijmans, H., Serra, J.: Convergence, continuity and iteration in mathematical morphology. Journal of Visual Communication and Image Representation 3, 84-102 (1992)

19. Jordens, O., Sturm, T.: Closure systems of equivalences with a local property. Mathematica Japonica 36(2), 245-250 (1991)

20. Jordens, O., Sturm, T.: Equivalences with closed equivalence classes. Mathematica Japonica 36(2), 291-304 (1991)

21. Lin, C.: The lattice of all partitions on a set. Tamkang J. Math. 4(2), 117-122 (1973)

22. Matheron, G.: Random Sets and Integral Geometry. J. Wiley and Sons, New York (1975)

23. Minkowski, H.: Volumen und Oberfläche. Math. Ann. 57, 447-495 (1903)

24. Mitchell, J.: Foundations for Programming Languages. MIT Press (1996)

25. Naegel, B., Passat, N., Ronse, C.: Grey-level hit-or-miss transforms - Part I: Unified theory. Pattern Recognition 40(2), 635-647 (2007)

26. Ore, O.: Theory of equivalence relations. Duke Mathematical Journal 9, 573-627 (1942)

27. Ronse, C.: Toggles of openings, and a new family of idempotent operators on partially ordered sets. Applicable Algebra in Engineering, Communication and Computing 3, 99-128 (1992)

28. Ronse, C.: A lattice-theoretical morphological view on template extraction in images. Journal of Visual Communication and Image Representation 7(3), 273-295 (1996)

29. Ronse, C.: Set-theoretical algebraic approaches to connectivity in continuous or digital spaces. Journal of Mathematical Imaging and Vision 8(1), 41-58 (1998)

30. Ronse, C.: Partial partitions, partial connections and connective segmentation. Journal of Mathematical Imaging and Vision 32(2), 97-125 (2008). DOI 10.1007/s10851-0080090-5

31. Ronse, C.: Adjunctions on the lattices of partitions and of partial partitions. Applicable Algebra in Engineering, Communication and Computing to appear (2010)

32. Ronse, C., Serra, J.: Fondements algébriques de la morphologie. In: L. Najman, H. Talbot (eds.) Morphologie Mathématique 1 - approches déterministes, chap. 2, pp. 49-96. Hermès / Lavoisier, Paris, France (2008)

33. Sabelfeld, A., Sands, D.: A per model of secure information flow in sequential programs. Higher-Order and Symbolic Computation 14(1), 59-91 (2001)

34. Serra, J.: Image Analysis and Mathematical Morphology. Academic Press, London (1982)

35. Serra, J. (ed.): Image Analysis and Mathematical Morphology, II: Theoretical Advances. Academic Press, London (1988)

36. Serra, J.: Mathematical morphology for Boolean lattices. In: J. Serra (ed.) Image Analysis and Mathematical Morphology, II: Theoretical Advances, chap. 2, pp. 37-58. Academic Press, London (1988)

37. Serra, J.: Connections for sets and functions. Fundamenta Informaticae 41(1/2), 147186 (2000)

38. Serra, J.: Morphological segmentations of colour images. In: C. Ronse, L. Najman E. Decencière (eds.) Mathematical Morphology: 40 Years On, Computational Imaging and Vision, vol. 30, pp. 151-176. Springer-Verlag, Dordrecht (2005)

39. Serra, J.: A lattice approach to image segmentation. Journal of Mathematical Imaging and Vision 24(1), 83-130 (2006)

40. Soille, P.: Morphological Image Analysis: Principles and Applications, 2nd edn. Springer, Berlin Heidelberg (2003)

41. Soille, P.: Constrained connectivity for hierarchical image partitioning and simplification. IEEE Transactions on Pattern Analysis and Machine Intelligence 30(7), 1132-1145 (2008)

42. Sturm, T.: Verbände von Kernen isotoner Abbildungen. Czekoslovak Mathematical Journal 22 (97), 126-144 (1972)

43. Tepavčević: Weak congruences in universal algebra. Tech. rep., Institute of Mathematics, Novi Sad (2001)

44. Vojvodić, G., Šešelja, B.: On the lattice of weak congruence relations. Algebra Universalis 25(1), 121-130 (1988) 
Table 2 Notation

\begin{tabular}{|c|c|}
\hline$E$ & space of points \\
\hline$T$ & set of image values (grey-levels or colours) \\
\hline $\mathrm{cr}$ & a criterion $T^{E} \times \mathcal{P}(E) \rightarrow\{0,1\}$ \\
\hline $\mathcal{C}_{\mathrm{cr}}^{F}$ & $\{A \in \mathcal{P}(E) \mid \operatorname{cr}[F, A]=1\}\left(F \in T^{E}\right)$ \\
\hline$(\alpha, \beta): A \rightleftharpoons B$ & $\alpha: A \rightarrow B$ and $\beta: B \rightarrow A$ \\
\hline $\ln v(\psi)$ & invariance domain of the operator $\psi$ \\
\hline $\operatorname{supp}(R)$ & support of the binary relation $R$ on $E$ \\
\hline $\operatorname{supp}(\mathcal{B})$ & support of the family $\mathcal{B}$ of subsets of $E$ \\
\hline$\pi$ & a partial partition \\
\hline $\operatorname{PE}(\pi)$ & partial equivalence corresponding to $\pi$ \\
\hline $\mathrm{Cl}_{\pi}$ & partial partition class map associated to $\pi$ \\
\hline$\Pi(E)$ & set of all partitions of $E$ \\
\hline$\Pi^{*}(E)$ & set of all partial partitions of $E$ \\
\hline$\varnothing$ & empty partial partition \\
\hline $\mathbf{0}_{A}$ & identity partition of $A$ into its singletons \\
\hline $\mathbf{1}_{A}$ & universal partition of $A$ into a single block \\
\hline 1. & $A \mapsto \mathbf{1}_{A}$ \\
\hline 0• & $A \mapsto \mathbf{0}_{A}$ \\
\hline $\mathcal{S}(E)$ & family of all singletons in $E$ \\
\hline $\mathcal{C}$ & a partial connection on $\mathcal{P}(E)$ \\
\hline$\gamma_{p}$ & partial connection opening on $\mathcal{P}(E)$ \\
\hline$\left(\gamma_{p}, p \in E\right)$ & system of partial connection openings on $\mathcal{P}(E)$ \\
\hline $\mathrm{PC}^{\mathcal{C}}(X)$ & partial partition of all $\mathcal{C}$-components of $X$ \\
\hline $\mathcal{C}_{s t d}$ & $\begin{array}{l}\text { a "standard" connection on } \mathcal{P}(E) \\
\text { (e.g., arc, topological, or graph connectivity) }\end{array}$ \\
\hline $\mathcal{C}_{B}^{*}$ & partial connection $\left\{Z \in \mathcal{C}_{s t d} \mid Z \circ B=Z\right\}\left(B \in \mathcal{C}_{s t d}, B \neq \emptyset\right)$ \\
\hline $\mathcal{C}_{B}$ & $\mathcal{C}_{B}^{*} \cup \mathcal{S}(E)$, connection generated by $\mathcal{C}_{B}^{*}$ \\
\hline $\mathcal{C}^{B}$ & connection $\left\{X \in \mathcal{P}(E) \mid X \oplus B \in \mathcal{C}_{s t d}\right\}\left(B \in \mathcal{C}_{s t d}, o \in B\right)$ \\
\hline$\Gamma(E)$ & set of all connections on $\mathcal{P}(E)$ \\
\hline$\Gamma^{*}(E)$ & set of all partial connections on $\mathcal{P}(E)$ \\
\hline $\operatorname{Con}(\mathcal{B})$ & connection generated by the family $\mathcal{B}$ \\
\hline $\operatorname{Con}^{*}(\mathcal{B})$ & partial connection generated by the family $\mathcal{B}$ \\
\hline$A E(L)$ & set of all anti-extensive operators on $L$ \\
\hline$A E^{I}(L)$ & set of all isotone anti-extensive operators on $L$ \\
\hline$[\pi]_{B}$ & $\pi \cap \mathcal{P}(B)$, for $B \in \pi^{*}, \pi \leq \pi^{*}$ \\
\hline$\sigma$ & set splitting operator $X \mapsto \sigma(X) \in \Pi^{*}(X)$ \\
\hline$\beta(\sigma)$ & block splitting operator on $\Pi^{*}(E)$ derived from $\sigma$ \\
\hline$\Sigma(E)$ & set of all set splitting operators \\
\hline$\beta^{+}, \beta^{-}$ & upper and lower adjoint of $\beta$ \\
\hline$\eta$ & anti-extensive operator on $\Pi^{*}(E)$ \\
\hline$\rho(\eta), \theta(\eta)$ & set splitting operators derived from $\eta$ \\
\hline$\Sigma^{I}(E)$ & set of all isotone set splitting operators \\
\hline $\mathbf{1}_{\psi}$ & set shrinking operator $X \mapsto \mathbf{1}_{\psi(X)}$ \\
\hline $\mathrm{B}(\psi)$ & $\pi \mapsto \bigvee_{B \in \pi} \mathbf{1}_{\psi(B)}$ \\
\hline$\Pi(E, \mathcal{C})$ & set of all partitions of $E$ with blocks in $\mathcal{C} \backslash\{\emptyset\}$ \\
\hline$\Pi^{*}(E, \mathcal{C})$ & set of all partial partitions of $E$ with blocks in $\mathcal{C} \backslash\{\emptyset\}$ \\
\hline $\operatorname{CS}^{\mathrm{C}}$ & opening on $\Pi^{*}(E)$ splitting blocks into $\mathcal{C}$-components \\
\hline $\mathcal{F}(\sigma)$ & $\left\{X \in \mathcal{P}(E) \mid \sigma(X)=\mathbf{1}_{X}\right\}$, fixed set of $\sigma$ \\
\hline $\operatorname{subbl}(\pi)$ & partial connection of all subsets of blocks of $\pi$ \\
\hline
\end{tabular}

